\title{
Electron correlation effects in electron-hole recombination in organic light-emitting diodes
}

\author{
Kunj Tandon, ${ }^{1 *}$ S. Ramasesha, ${ }^{1}$ and S. Mazumdar ${ }^{2}$ \\ ${ }^{1}$ Solid State and Structural Chemistry Unit, Indian Institute of Science, Bangalore 560 012, India \\ ${ }^{2}$ Department of Physics and The Optical Sciences Center, University of Arizona, Tucson, Arizona 08721
}

\begin{abstract}
We develop a general theory of electron-hole recombination in organic light-emitting diodes that leads to formation of emissive singlet excitons and nonemissive triplet excitons. We briefly review other existing theories and show how our approach is substantively different from these theories. Using an exact timedependent approach to the interchain/intermolecular charge transfer within a long-range interacting model we find that (i) the relative yield of the singlet exciton in polymers is considerably larger than the $25 \%$ predicted from statistical considerations, (ii) the singlet exciton yield increases with chain length in oligomers, and (iii) in small molecules containing nitrogen heteroatoms, the relative yield of the singlet exciton is considerably smaller and may be even close to $25 \%$. The above results are independent of whether or not the bond-charge repulsion, $X_{\perp}$, is included in the interchain part of the Hamiltonian for the two-chain system. The larger (smaller) yield of the singlet (triplet) exciton in carbon-based long-chain polymers is a consequence of both its ionic (covalent) nature and smaller (larger) binding energy. In nitrogen containing monomers, wave functions are closer to the noninteracting limit, and this decreases (increases) the relative yield of the singlet (triplet) exciton. Our results are in qualitative agreement with electroluminescence experiments involving both molecular and polymeric light emitters. The time-dependent approach developed here for describing intermolecular charge-transfer processes is completely general and may be applied to many other such processes.
\end{abstract}

\section{INTRODUCTION}

Charge recombination and photoinduced charge transfer lie at the heart of current attempts to construct viable optoelectronic devices using organic semiconducting materials consisting of $\pi$-conjugated polymers or molecules. Charge recombination is the fundamental process of interest in organic light-emitting diodes (OLED's). Electroluminescence (EL) in OLED's results from (a) the injection of electrons and holes into thin films containing the emissive material, (b) migration of these charges, which can involve both coherent motion on a single chain and interchain or intermolecular charge transfer between neutral and charged species, (c) recombination of electrons and holes on the same polymer chain or molecule. ${ }^{1-3}$ If the recombination leads to the singlet optical exciton, light emission can occur. If, on the other hand, the final product of the recombination is a triplet exciton, only nonradiative relaxation can occur in the absence of strong spin-orbit coupling. EL in OLED's is of strong current interest, both because of applications in display devices ${ }^{4,5}$ and the potential for obtaining organic solid state lasers. The fundamental process that occurs in photoinduced charge transfer is the exact reverse of that in EL: optical excitation to the singlet exciton in a donor molecule is followed by charge separation and migration of charge to a neighboring acceptor molecule. The latter process is of interest in photovoltaic applications. ${ }^{6}$

The fundamental electronic process of charge recombination or separation is therefore of strong current interest. Especially in the context of EL in OLED's, charge recombination has received both experimental and theoretical attention (see below). The overall quantum efficiency of the EL depends on (i) the fraction of the total number of injected carriers that end up as excitons on the same polymeric chain or molecule, (ii) the fraction of these excitons that are spin singlets, since only singlet excitons are emissive, and (iii) the fraction of singlet excitons that actually undergo radiative decay. In the present paper we focus on (ii), which determines the maximum possible EL efficiency.

Formally, the charge recombination process can be written as

$$
P^{+}+P^{-} \rightarrow G+S / T
$$

where $P^{ \pm}$are charged polaronic states of the emissive molecule, $G$ is the ground state of the neutral molecule, and $S$ and $T$ are singlet and triplet excited states of the neutral molecule. Equation (1) indicates that both singlet and triplet excitons are likely products of the charge recombination process. We shall denote the fraction of singlet excitons generated in OLED's by the above recombination process as $\eta$.

Early discussions of $\eta$ were based on statistical arguments alone. Since electrons and holes are injected independently from the two electrodes, and since two spin-1/2 particles can give three independent spin 1 states (with $M_{S}=-1,0$, and $+1)$ but only one spin 0 state $\left(M_{S}=0\right)$, it follows that $\eta$ is 0.25 . Note, however, that this argument is strictly valid only for noninteracting electrons, such that single-configuration molecular orbital descriptions of all eigenstates are valid. In such a case, the highest occupied and lowest unoccupied molecular orbitals (HOMO and LUMO) are identical for the singlet and triplet excited states. Charge recombination [Eq. (1)] then involves merely the migration of an electron from the doubly (singly) occupied HOMO (LUMO) of $P^{-}$to the singly occupied (unoccupied) HOMO (LUMO) of the $P^{+}$, for both singlet and triplet channels. The singlet channel and all three triplet channels of the charge recombination process are equally likely within the MO scheme. If electrons are 
interacting, however, this simple single-configuration description breaks down, as all the states included in Eq. (1) are now superpositions of multiple configurations. There is no longer any fundamental reason for the singlet and triplet channels to be equally likely processes, and hence there is no reason for $\eta$ to be 0.25 .

Experimentally, $\eta$ has been found to range from $\sim 0.25$ to 0.66 (Refs. 7-10) in different materials. In OLED's with the molecular species aluminum tris (8-hydroxyquinoline) $\left(\mathrm{Alq}_{3}\right)$ as the emissive material Baldo et al. have determined $\eta \sim 0.22 \pm 0.03$, in agreement with that expected from statistical arguments. ${ }^{7}$ On the other hand, considerably larger $\eta$ $\sim 0.45$ has been found in derivatives of poly(paraphenylenevinylene) (PPV) by Cao et al. and Ho et al., Wohlgenannt et al., using spin-dependent recombination spectroscopy, have determined the formation cross sections of singlet and triplet excitons, $\sigma_{S}$ and $\sigma_{T}$, respectively, for a large number of polymeric materials (including nonemissive polymers in which the lowest two-photon state $2 A_{g}$ occurs below the optical $1 B_{u}$ exciton), and found that $\sigma_{S} / \sigma_{T}$ is strongly material dependent and in all cases considerably larger than 1 (thereby implying that $\eta$ is material dependent and much larger than 0.25). ${ }^{10}$ More recently, Wilson et al. ${ }^{11}$ and Wohlgenannt et al. ${ }^{12}$ have shown that $\eta$ can depend strongly on the effective conjugation length, with values ranging from $\sim 0.25$ for small monomers to considerably larger than 0.25 for long chain oligomers.

Theoretically, $\eta$ has been investigated by a number of groups $^{10,13-16}$ including ourselves. There is general agreement that $\eta$ can be substantially greater than 0.25 in $\pi$-conjugated polymers and that this is an electron correlation effect. There exist, however, substantial differences between the assumptions and formalisms that go into these theories. The goal of the present work is to develop a formalism that gives a clear physical picture of the electronhole recombination and explains why $\eta$ substantially larger than 0.25 is to be expected in organic polymeric systems. Ideally, since photoinduced charge transfer is the exact reverse process of electron-hole recombination, it should also be possible to extend our approach to photoinduced charge transfer in the future. A brief presentation of our work was made earlier, ${ }^{10}$ where, however, the emphasis was more on the experimental technique used by our experimental collaborators. Here we present the full theoretical details of our earlier work, provide a critique of the earlier theories and also report on the new and interesting results of our investigation of external electric field effects on $\eta$, albeit for artificially large fields, and also on the role of nitrogen heteratoms in electron-hole recombination. Specifically, our theoretical approach involves a time-dependent formalism, within which the initial state composed of two oppositely charged polarons is allowed to propagate in time under the influence of the complete Hamiltonian that includes both onchain and interchain interactions. For the sake of completeness, we also discuss other existing theoretical approaches, ${ }^{13-16}$ and their applicability to real systems. In particular, there exists a superficial similarity between the approach used in Refs. 15 and 16 and ours. For a physical understanding of the electron-hole recombination process it is essential (see below) that the difference between our approach and that used by the authors of Refs. 15 and 16 is precisely understood.

The plan of the paper is as follows. In Sec. II, we present our theoretical models for intrachain and interchain interactions, and also discuss the model systems that are studied. In Sec. III we present a brief critique of the existing theories. A more extended discussion of the approach used by Shuai and co-workers $^{15,16}$ is given in Appendix A. In Sec. IV we present the method of propagation of the initial state, while in Sec. V we present our numerical results. In this section we also discuss an alternate approach to the time propagation for the simplest case of two ethylenes that confirms the validity of the more general approach used in Sec. IV, and that also gives a physical picture of the recombination process. While $\eta>0.25$ is found in our calculations with interacting electrons, the absolute yields of both singlet and triplet excitons are found to be extremely small with standard electron correlation parameters. We therefore investigate the effects of the external electric field on these yields within a highly simplified model. It is found that for sufficiently large fields the yields with interacting electrons are as large as those with noninteracting electrons in the field-free case, and that in the relatively small field region $\eta$ continues to be greater than 0.25 . In the very high field regime it is found that $\eta$ can even be smaller than 0.25 . While the bare electric fields required to see the reversal of the singlet-triplet ratio are rather large and therefore only of academic interest, if internal field effects are taken into account, it is possible to envisage situations where the effective electric field is large enough to bring about such a reversal in the singlet-triplet ratio. Following the discussion of electric field effects, we discuss how the chain-length dependence of $\eta$, as observed experimentally, ${ }^{11,12}$ can be understood within our theory. We then consider the role of heteroatoms, especially in the context of molecular emitters. We show that in small systems with heteroatoms $\eta$ can approach the statistical limit, thus explaining qualitatively the monomer results of Wilson et al., ${ }^{11}$ and the results of Baldo et al. for $\mathrm{Alq}_{3} .{ }^{7}$ The emphasis in all our calculations is on understanding the qualitative aspects of charge recombination and not on detailed quantitative aspects. Finally in Sec. VI we discuss the conclusions and scope of future work.

\section{THEORETICAL MODEL}

The goal of the present work is to provide benchmark results for the charge recombination reaction which are valid for the strong Coulomb interactions that characterize $\pi$-conjugated systems. Accurate treatments of electronelectron interactions are not possible for long-chain systems, and in this initial study we have therefore chosen pairs of short polyene chains, with 2-6 carbon atoms in each chain as our model systems. Since polyene eigenstates possess mirror-plane and inversion symmetries, we shall henceforth refer to the ground state $G$ [see Eq. (1)] as $1{ }^{1} A_{g}$, and $S$ and $T$ as $1{ }^{1} B_{u}$ and $1{ }^{3} B_{u}$, respectively. The model system containing two hexatrienes (12 carbon atoms overall) is the larg- 
est system that can be treated exactly at present within correlated electron models.

Our approach suffers from two apparent disadvantages. First, polyenes and polyacetylenes are weakly emissive because the $2{ }^{1} A_{g}$ state in these occur below the optical $1{ }^{1} B_{u}$ state. This presents no problem as far as the analysis of the EL in emissive materials is concerned, as the spectroscopic technique of Wohlgenannt et al. ${ }^{10}$ finds a strong deviation of $\sigma_{S} / \sigma_{T}$ from 1 even in systems with energy ordering similar to that in polyenes ${ }^{10}$ [see results for Poly-2,5-Thienylene Vinylene (PTV) in this paper, for instance], and as we show in the following, this is a direct consequence of the large energy difference between the singlet $1{ }^{1} B_{u}$ exciton and the triplet $1{ }^{3} B_{u}$ exciton, as well as the fundamental difference in their electronic structures. Both, in turn, are consequences of strong electron-electron interactions, which also characterize systems like PPV and poly-paraphenylene (PPP), as evidenced from the large difference in energies between the singlet and triplet excitons in these systems, determined experimentally, ${ }^{17-20}$ as well as theoretically. ${ }^{21,22}$ A second apparent disadvantage of our procedure is related to the limitation of our calculations to short systems. This prevents direct evaluation of the chain-length dependence of $\eta$. We believe that this problem can be circumvented once the mechanism of the physical process that leads to the difference between singlet and triplet generation is precisely understood, and for this purpose it is essential that the electron correlation effects are investigated thoroughly using exactly solvable models. As we show later, our approach gives a precise though qualitative explanation of the chain length dependence.

Our model system consists of two polyene chains of equal lengths that lie directly on top of each other, separated by $4 \AA$. We consider the charge recombination process of Eq. (1), and there are two possible initial states: (i) a specific chain (say chain 1) is positively charged, with the other (chain 2) have negatively charged, a configuration that hereafter we denote as $P_{1}^{+} P_{2}^{-}$, where the subscripts 1 and 2 are chain indices, or (ii) the superposition $P_{1}^{+} P_{2}^{-} \pm P_{2}^{+} P_{1}^{-}$, in the same notation. In our calculations we have chosen the first as the proper initial state, since experimentally in the OLED's the symmetry between the chains is broken by the external electric field (we emphasize that the consequence of choosing the symmetric or antisymmetric superposition can be easily predicted from our all our numerical calculations that follow). Even with initial state (i), the final state can consist of both $\left(1^{1} A_{g}\right)_{1}\left(1^{1} B_{u}\right)_{2}$ and $\left(1{ }^{1} A_{g}\right)_{2}\left(1{ }^{1} B_{u}\right)_{1}$ in the singlet channel. The same is true in the triplet channel, i.e., either of the two chains can be in the ground (excited) state. Hereafter we will write the initial states as $\left|i_{S}\right\rangle$ and $\left|i_{T}\right\rangle$, where the subscripts $S$ and $T$ correspond to spin states $S$ $=0$ and 1 . We consider only the $M_{S}=0$ triplet state. The initial states are simply the product states with appropriate spin combinations,

$$
\begin{aligned}
& \left|i_{S}\right\rangle=2^{-1 / 2}\left(\left|P_{1, \uparrow}^{+}\right\rangle\left|P_{2, \downarrow}^{-}\right\rangle-\left|P_{1, \downarrow}^{+}\right\rangle\left|P_{2, \uparrow}^{-}\right\rangle\right), \\
& \left|i_{T}\right\rangle=2^{-1 / 2}\left(\left|P_{1, \uparrow}^{+}\right\rangle\left|P_{2, \downarrow}^{-}\right\rangle+\left|P_{1, \downarrow}^{+}\right\rangle\left|P_{2, \uparrow}^{-}\right\rangle\right) .
\end{aligned}
$$

There exist of course two other initial triplet states with $M_{S}= \pm 1$. The overall Hamiltonian for our composite twochain system consists of an intrachain terms $H_{\text {intra }}$ and interchain interactions $H_{\text {inter }}$. Additional interactions must be explicitly included to discuss external influences like the electric field, etc. $H_{\text {intra }}$ describing individual chains is the Pariser-Parr-Pople (PPP) Hamiltonian ${ }^{23,24}$ for $\pi$-electron systems, written as

$$
\begin{aligned}
H_{\text {intra }}= & -\sum_{\langle i j\rangle, \sigma} t_{i j}\left(a_{i, \sigma}^{\dagger} a_{j, \sigma}+\text { H.c. }\right)+\sum_{i} \epsilon_{i} n_{i} \\
& +\sum_{i} U_{i} n_{i, \uparrow} n_{i, \downarrow}+\sum_{i>j} V_{i j}\left(n_{i}-z_{i}\right)\left(n_{j}-z_{j}\right),
\end{aligned}
$$

where $a_{i, \sigma}^{\dagger}$ creates a $\pi$ electron of spin $\sigma$ on carbon atom $i, n_{i, \sigma}=a_{i, \sigma}^{\dagger} a_{i, \sigma}$ is the number of electrons on atom $i$ with spin $\sigma$ and $n_{i}=\Sigma_{\sigma} n_{i, \sigma}$ is the total number of electrons on atom $i, \epsilon_{i}$ is the site energy, and $z_{i}$ are the local chemical potentials. The hopping matrix element $t_{i j}$ in the above are restricted to nearest neighbors and in principle can contain electron-phonon interactions, although a rigid bond approximation is used here. $U_{i}$ and $V_{i j}$ are the on-site and intrachain intersite Coulomb interactions.

We use standard parametrizations for $H_{\text {intra }}$. The hopping integrals for single and double bonds are taken to be 2.232 and $2.568 \mathrm{eV}$, respectively, and all the site energies of carbon atoms in a polymer with all equivalent sites are set to zero. We choose the Hubbard interaction parameter $U_{C}$ for carbon to be $11.26 \mathrm{eV}$, and for the $V_{i j}$ we choose the Ohno parametrization, ${ }^{25}$

$$
V_{i j}=14.397\left[\left(\frac{28.794}{U_{i}+U_{j}}\right)^{2}+r_{i j}^{2}\right]^{-1 / 2},
$$

where the distance $r_{i j}$ is in $\AA, V_{i j}$ is in $\mathrm{eV}$ and the local chemical potential $z_{C}$ for $s p^{2}$ carbon is one. It should be noted then when heteroatoms such as nitrogen are present, the on-site correlation energy, the site energy, and the local chemical potential could be different from those for carbon. For $H_{\text {inter }}$, we choose the following form:

$$
\begin{aligned}
H_{\text {inter }}= & -t_{\perp} \sum_{i, \sigma}\left(a_{i \sigma}^{\dagger} a_{i, \sigma}^{\prime}+\text { H.c. }\right) \\
& +X_{\perp} \sum_{i, \sigma}\left(n_{i}+n_{i}^{\prime}\right)\left(a_{i \sigma}^{\dagger} a_{i, \sigma}^{\prime}+\text { H.c. }\right) \\
& +\sum_{i, j} V_{i, j}\left(n_{i}-z_{i}\right)\left(n_{j}^{\prime}-z_{j^{\prime}}\right) .
\end{aligned}
$$

In the above, primed and unprimed operators correspond to sites on different chains. Note that the interchain hopping $t_{\perp}$ is restricted to corresponding sites on the two chains, which are nearest interchain neighbors. The interchain Coulomb interaction $V_{i, j}$, however, includes interaction between any site on one chain with any other site on the other chain. In addition to the usual one-electron hopping that occurs within the zero differential overlap approximation ${ }^{23,24}$ we have also included a many-electron site charge-bond charge repulsion $X_{\perp}$ (operating between nearest interchain neighbors only) 
that consists of multicenter Coulomb integrals. This term should also occur within $H_{\text {intra }}$, but is usually ignored there because of its small magnitude, relative to all other terms. ${ }^{23,24,26}$ In contrast, the $t_{\perp}$ in $H_{\text {inter }}$ is expected to be much smaller, and $X_{\perp}$ cannot be ignored in interchain processes, especially at large interchain separations. ${ }^{27} \mathrm{We}$ have done calculations for both $X_{\perp}=0$ and $X_{\perp} \neq 0$.

\section{BRIEF CRITIQUE OF EXISTING THEORIES}

To put our work in the proper context we present a discussion of the existing theories of charge recombination ${ }^{13-16}$ in this section. The natures of $H_{\text {intra }}$ within all these models are similar in the sense that they all incorporate intrachain Coulomb interactions, without which of course there cannot be any difference between singlet and triplet generation. Following this, there is a fundamental difference between the models of Refs. 13 and 14 on the one hand, and those of Refs. 15 and 16 and ours on the other. Within the theory of Refs. 13 and 14, there is no difference in singlet or triplet generation in the first stage of the charge-recombination process, which involves interchain charge transfer. Within these models, interchain charge-transfer yields high energy singlet and triplet excited states of long chains that occur in the continuum, and the lowest singlet and triplet excitons result from relaxations of these high energy states. Differences in the relative yields of the lowest singlet and triplet excitons are consequences of differences in the intrachain relaxation processes in the singlet and triplet channels, which occur in the second stage of the overall process. In contrast, within our theory ${ }^{10}$ and the theory of Refs. 15 and 16, the lowest singlet and triplet excitons are generated directly from two oppositely charged polarons, and their different yields are consequences of the different cross sections of the interchain charge-transfer reactions in the singlet and triplet channels.

Within the model of Hong and Meng, ${ }^{13}$ the continuum singlet state decays to the lowest singlet exciton, while the continuum triplet state decays to a high energy triplet state $T_{2}$ consisting of a loosely bound triplet exciton, which then relaxes nonradiatively to the lowest tightly bound triplet exciton $T_{1}$. The energy gap between $T_{2}$ and $T_{1}$ is large, and according to Hong and Meng, this nonradiative relaxation has to be a multiphonon cascade process. The large energy gap and the multiphonon nature of the relaxation creates a "bottleneck" in the $T_{2} \rightarrow T_{1}$ nonradiative transition, and spin-orbit coupling leads to intersystem crossing from $T_{2}$ to the singlet exciton, thereby increasing the relative yield of singlets. ${ }^{13}$ We believe that the key problem with this approach is that the model is in disagreement with what is known about the spectrum of triplet states from triplet absorptions in $\pi$-conjugated polymers ${ }^{19}$ and theoretical solutions to the PPP model. ${ }^{22}$ Experimentally, in PPV, for instance, the lowest triplet occurs at about $1.55 \mathrm{eV},{ }^{19}$ while in MEH-PPV this state occurs at $\sim 1.3 \mathrm{eV} .{ }^{17}$ The triplet absorption energy in these systems is about $1.4 \mathrm{eV}$. Theoretically, the final state in triplet absorption occurs slightly below the continuum band, ${ }^{22}$ and this is therefore the $T_{2}$ state [also referred to as the $m^{3} A_{g}$ (Ref. 19)]. The energy region between $T_{2}$ and $T_{1}\left(m^{3} A_{g}\right.$ and $\left.1^{3} B_{u}\right)$ in the triplet subspace is not at all sparse, as assumed by Hong and Meng, but rather, within the correlated PPP Hamiltonian $H_{\text {intra }}$ in Eq. (4), this energy region contains numerous other triplet states. ${ }^{28,29}$ Thus any nonradiative relaxation from $T_{2}$ to $T_{1}$ in the realistic systems should involve a number of intermediate triplet states with small energy gaps between them, and therefore the phonon bottleneck simply will not occur. An additional problem with the model of Hong and Meng is that even in the singlet channel, generation of the lowest exciton from a continuum singlet state cannot be direct but can occur only through the $m^{1} A_{g}$ loosely bound singlet exciton. ${ }^{22}$ In principle, this can lead to a bottleneck even in the singlet channel. To summarize, we believe that the model of Hong and Meng is in disagreement with the known singlet and triplet energy spectra within the PPP model.

Within the model of Kobrak and Bittner ${ }^{14}$ also polaron pairs are formed on the single chain first. These authors take into account the electron-phonon interactions explicitly, and the two-particle states on a single chain are allowed to evolve by interacting with a one-dimensional classical vibrational lattice. Different cross sections for singlet and triplet excitons are found within the authors' model, and the difference originates from the difference in the mixing between the polaron and exciton states with different spin. The theory includes only the Coulomb interactions between the polaron charges and not the Coulomb interactions between all the electrons that appear in the PPP Hamiltonian. The theory also assumes large quantum efficiency for the generation of the high energy states with the two polaron charges on the same chain, starting from a state with the charges on different chains. A recent calculation by Ye et al. ${ }^{16}$ indicates very weak cross sections for the generation of high energy ${ }^{1} B_{u}$ and ${ }^{3} B_{u}$ states starting from the initial state containing the charges on different chains (see Fig. 8 in Ref. 16). This is supported also by our exact calculations (see below). However, the calculations by Ye et al. ${ }^{16}$ as well as ours are for relatively short chains, and further work is needed to test the validity of the model of Kobrak and Bittner. As we show in Sec. V, $\eta>0.25$ is predicted from considerations of the initial stage of interchain charge-transfer alone. Whether additional contributions can come from differences in the intrachain relaxation processes needs to be studied further.

We now come to the work by Shuai and co-workers, ${ }^{15,16}$ who, like us, have determined $\eta>0.25$ in oligomers of PPV from considerations of interchain charge transfer. Precisely because of the apparent similarity of our approaches, it is essential that we discuss the approach of Shuai and coworkers in detail, since our ultimate goal is to arrive at a physical explanation of the greater yield of the singlet exciton than what is predicted from statistical considerations, and as we show later, the physical mechanisms within Refs. 15 and 16 and within our work are quite different. The quantity that is calculated in Refs. 15 and 16 is $\sigma_{S} / \sigma_{T}$, viz., the ratio of the formation cross sections of the $1{ }^{1} B_{u}$ singlet and $1{ }^{3} B_{u}$ triplet exciton. For fast spin-lattice interaction, the expression for $\eta$ in terms of $\sigma_{S}$ and $\sigma_{T}$ can be written as ${ }^{10,12}$

$$
\eta=\sigma_{S} /\left(\sigma_{S}+3 \sigma_{T}\right)
$$

and thus for $\sigma_{S} / \sigma_{T}>1, \quad \eta>0.25$. 
Shuai et al. consider the same $H_{\text {intra }}$ as us, and $H_{\text {inter }}$ that is similar (see below). The authors then use the Fermi "golden rule" approach to calculate $\sigma_{S}$ and $\sigma_{T}$. According to the authors, the cross-section ratio is given by

$$
\sigma_{S} / \sigma_{T}=\left|\left\langle i_{S}\left|H_{\text {inter }}\right| f_{S}\right\rangle\right|^{2} /\left|\left\langle i_{T}\left|H_{\text {inter }}\right| f_{T}\right\rangle\right|^{2},
$$

where $\left|i_{S}\right\rangle$ and $\left|i_{T}\right\rangle$ are the singlet and triplet initial states [see Eqs. (2) and (3)], and $\left|f_{S}\right\rangle$ and $\left|f_{T}\right\rangle$ are the corresponding final states, respectively. Since the interchain Coulomb interaction is diagonal in the space of the states considered in Eq. (8), the authors ignore $V_{i, j}$ in Eq. (6) but retain the other terms. Shuai and co-workers find that for $X_{\perp}=0$ in Eq. (6), when the interchain charge transfer is due to the hopping $t_{\perp}$ only, the right-hand side of Eq. (8) is $\sim 1$, a result we agree with (see Appendix A). The authors then claim that for nonzero positive $X_{\perp}$, and for positive $t_{\perp}$ [note negative sign in front of the one-electron term in Eq. (6)], the right-hand side of Eq. (8) can be substantially larger than 1. The authors calculated the matrix elements in Eq. (8) for pairs of PPV oligomers in parallel configuration using approximate methods (singles configuration interaction ${ }^{15}$ and coupled-cluster $\operatorname{method}^{16}$ ), and have found the right-hand side of Eq. (8) to show divergent behavior over a broad range of $X_{\perp} / t_{\perp}$ (see Fig. 1 in Ref. 15 and Figs. 3, 4, 6, and 7 in Ref. 16). Based on these calculations the authors conclude that a moderate to large $X_{\perp}$ is essential for the experimentally observed large $\sigma_{S} / \sigma_{T}{ }^{\overline{8}-12}$

This result is surprising, in view of the fact that the site charge-bond charge repulsion is spin-independent, exactly as the one-electron interchain hopping in Eq. (6). Since this question is intimately linked with the mechanism of charge recombination that we are after we have reexamined this issue by performing exact calculations for pairs of polyene chains with lengths $N=2,4$, and 6 . The conclusions from these exact calculations are described below.

As discussed above, even with $P_{1}^{+} P_{2}^{-}$as the initial state (with, of course, appropriate spin functions) the final state contains two terms, with one of the two chains in the ground state and the other in the excited state. Instead of working with different superpositions of the final states we consider $\sigma_{S}$ to be proportional to $\left|\left\langle i_{S}\left|H_{\text {inter }}\right|\left(1^{1} A_{g}\right)_{1}\left(1{ }^{1} B_{u}\right)_{2}\right\rangle\right|^{2}$ $+\left|\left\langle i_{S}\left|H_{\text {inter }}\right|\left(1^{1} A_{g}\right)_{2}\left(1^{1} B_{u}\right)_{1}\right\rangle\right|^{2}$. Similarly, $\sigma_{T}$ is taken to be proportional to $\left|\left\langle i_{T}\left|H_{\text {inter }}\right|\left(1^{1} A_{g}\right)_{1}\left(1^{3} B_{u}\right)_{2}\right\rangle\right|^{2}$ $+\left|\left\langle i_{T}\left|H_{\text {inter }}\right|\left(1{ }^{1} A_{g}\right)_{2}\left(1{ }^{3} B_{u}\right)_{1}\right\rangle\right|^{2}$. As shown explicitly in Appendix $A$, the magnitudes of the matrix elements of the initial singlet [triplet] $P_{1}^{+} P_{2}^{-}$with $\left(1^{1} A_{g}\right)_{1}\left(1^{1} B_{u}\right)_{2}\left[\left(1^{1} A_{g}\right)_{1}\left(1^{3} B_{u}\right)_{2}\right]$ and $\left(1^{1} A_{g}\right)_{2}\left(1^{1} B_{u}\right)_{1}$ $\left[\left(1^{1} A_{g}\right)_{2}\left(1^{3} B_{u}\right)_{1}\right]$ are different for $X_{\perp} \neq 0$, and hence the final states cannot be 1:1 superpositions of these configurations. Note that by taking the sums of the squares we exhaust all possibilities automatically. For the conclusions of Refs. 15 and 16 to be valid the calculated $\sigma_{S} / \sigma_{T}$ within Eq. (8) should now show strong dependence on $X_{\perp} / t_{\perp}$ (as mentioned above divergent $\sigma_{S} / \sigma_{T}$ is implied in Refs. 15 and 16). Our exact results for the three different chain lengths are shown in Fig. 1 below, where we see that only for $X_{\perp} / t_{\perp}$ very close to 0.5 is $\sigma_{S} / \sigma_{T}$, as calculated within Eq. (8), is substantially different from 1 . At all other $X_{\perp} / t_{\perp}$ the right-

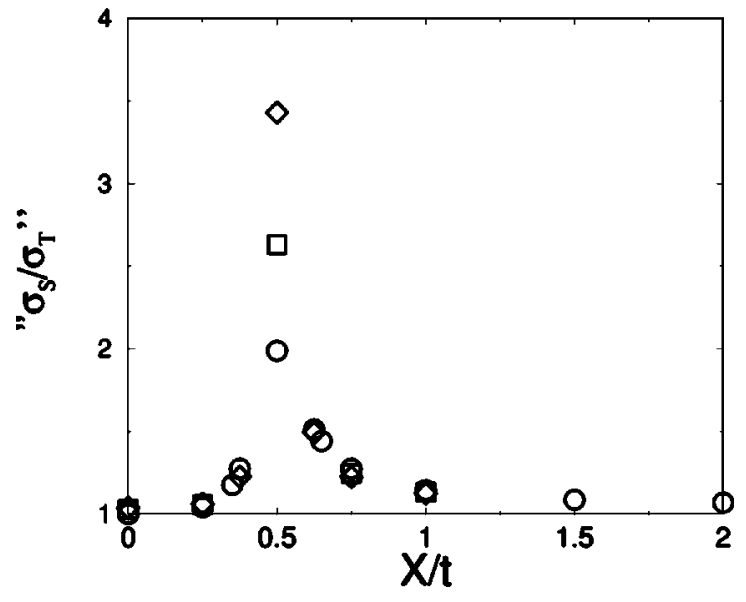

FIG. 1. The ratio of the squares of the singlet and triplet matrix elements of $H_{\text {inter }}$ [ $\sigma_{S} / \sigma_{T}$ according to Eq. (8)], as a function of $X_{\perp} / t_{\perp}$ for pairs of ethylenes (circles), butadienes (squares) and hexatrienes (diamonds).

hand side of Eq. (8) is very close to 1 . Furthermore, except for $X_{\perp} / t_{\perp}=0.5$ the chain-length-dependence of $\sigma_{S} / \sigma_{T}$ is weak. If we now recall that all chain length dependent quantities (for example, optical and other energy gaps in polyenes ${ }^{28}$ ) exhibit strongest length dependence at the shortest lengths, the conclusion that emerges is that except for the unique point $X_{\perp} / t_{\perp}=0.5, \sigma_{S} / \sigma_{T}$ remains $\sim 1$ within the golden rule approach even in the long chain limit.

In order to understand this difference from the results of Shuai and co-workers ${ }^{15,16}$ in further detail we present analytic results for the case of two ethylenes $(N=2)$ in Appen$\operatorname{dix}$ A. These results are important in so far as they begin to give a physical picture for the charge recombination reaction, even as they indicate that the site charge-bond charge repulsion is not the origin of large $\eta$. The analytic calculations also make the origin of the uniqueness of the point $X_{\perp} / t_{\perp}$ $=0.5$ absolutely clear. Indeed it is seen that precisely at this point both $\sigma_{S}$ and $\sigma_{T}$, as defined in Eq. (8), approach zero. More importantly, the chain length independence, as suggested in Fig. 1 can be understood very clearly from the analytic calculations. Finally, it can also be seen from these calculations that had we taken the initial state to be the superposition $P_{1}^{+} P_{2}^{-} \pm P_{2}^{+} P_{1}^{-}$, instead of only one of these, the $\sigma_{S} / \sigma_{T}$, as calculated from Eq. (8) would be exactly 1 for all $X_{\perp} / t_{\perp}$.

Our basic conclusion then is that the Fermi golden rule approach is not valid for calculations of $\sigma_{S} / \sigma_{T}$ or $\eta$. This is to be expected also from a different consideration, viz., the Fermi golden rule approach is valid for calculations of states that lie within a narrow band, whereas in the present case the energy difference between the initial and final states, and that between the singlet and triplet excitons are both much larger than $t_{\perp}$ and $X_{\perp}$. The origin of the difference between our exact calculations of matrix elements and the approximate calculations of Shuai et al. is harder to ascertain. One possibility is that the polaron wave functions are open shell, and approximating these within mean field or limited configuration interaction $(\mathrm{CI})$ could lead to wrong conclusions. In the 
following sections we therefore go beyond the Fermi golden rule approach to understand the origin of large $\eta$.

\section{TIME EVOLUTION OF THE POLARON PAIR STATE}

A straightforward numerical solution of $H_{\text {intra }}+H_{\text {inter }}$ will give all eigenfunctions of the composite two-chain systems, as linear superpositions of product eigenstates of the single chains (for example, $a\left|1{ }^{1} A_{g}\right\rangle_{1}\left|1{ }^{1} A_{g}\right\rangle_{2}+b\left|1{ }^{1} A_{g}\right\rangle_{2}\left|1{ }^{2} A_{g}\right\rangle_{1}$ $+\cdots$, etc., where $a, b$, etc. are relative weights). Several of the excited states can be superpositions of $\left|P_{1}^{+} P_{2}^{-}\right\rangle$and $\left|1{ }^{1} A_{g}\right\rangle_{1}\left|1{ }^{1} B_{u}\right\rangle_{2}$ or $\left|1{ }^{1} A_{g}\right\rangle_{1}\left|1{ }^{3} B_{u}\right\rangle_{2}$, but not only is it not clear how to obtain information on the relative yields of singlets and triplets in OLED's from such calculations, the search for all such states through the complete energy space would be extremely difficult if not impossible. Recall, for example, that (i) excited states higher in energy than $1{ }^{1} B_{u}$ or $1^{3} B_{u}$ can also in principle contribute to the overall singlet and triplet yield, (ii) the total number of excited states of the composite two-chain system is very large, and (iii) the quantum numbers of the excited states that are appropriate superpositions are not known in advance, and thus a methodical state-by-state search would have to be carried out for our purpose.

Our approach therefore consists in calculating the time evolution of the initial states $\left|i_{S}\right\rangle$ and $\left|i_{T}\right\rangle$ [see Eqs. (2) and (3)] under the influence of the full Hamiltonian, and then evaluating the overlaps of the time-evolved states with all possible final states $\left|f_{S}\right\rangle$ and $\left|f_{T}\right\rangle$. Our choice of initial states is thus the same as those in Refs. 15 and 16, but the difference lies in our going beyond the golden rule approximation to calculate the time evolution. The choice of an eigenstate of the unperturbed Hamiltonian as an initial state in a timedependent calculation, even when the perturbation itself is not explicitly time-dependent, is an approximation which is widely used - for example in collision theory (see Chaps. 18 and 19 in Ref. 30) or in many-body calculations of the dielectric function ${ }^{31}$-and is analogous to the sudden approximation. Specifically in the OLED's, the $P^{ \pm}$are created at opposite ends of the device and they execute hopping motion towards each other under the influence of an external electric field $\left(P^{ \pm}+G \rightarrow G+P^{ \pm}\right)$. The polaron wave functions remain unperturbed throughout this process, until they are within the radius of influence of each other. It is at this time $t=0$ we visualize that the interchain interactions are "switched on" suddenly from zero, and the intermolecular charge-transfer hereafter is rapid (several to several tens of femtoseconds, for realistic interchain hopping $t_{\perp}$, see below). It is the ultrashort time scale of this charge-transfer process that justifies the choice of the initial state. Finally, we reemphasize that as already mentioned in Sec. I, the goal of our study is to obtain qualitative but clear mechanistic description of the charge-transfer process, and this is best obtained with the well-defined charged-polaron pair states as the initial states.

In principle, given a Hamiltonian, propagation of any initial state is easily achieved by solving the time-dependent Schrödinger equation. One could use the interaction picture to separate the nontrivial evolution of the initial state from the trivial component, which occurs as a result of the evolution of the product of the eigenstates of the Hamiltonian of the subsystems. ${ }^{32}$ In the context of the many-body PPP Hamiltonian such an approach is difficult to implement numerically. This is because the total number of eigenstates for the two-chain system is very large: the number of such states for two chains of six carbon atoms each is 853776 in the $M_{s}=0$ subspace. Obtaining all the eigenstates of the twocomponent system and expressing the matrix elements of $H_{\text {inter }}$ in the basis of these eigenstates is therefore very intensive computationally. It is simpler to calculate the time evolution in the Schrodinger representation, determine the timeevolved states, and project them on to the desired final eigenstates (for instance, $\left|1{ }^{1} A_{g}\right\rangle_{1}\left|1^{1} B_{u}\right\rangle_{2}$ ). This is the approach we take.

We first obtain the eigenstates $\left|P_{1}^{+}\right\rangle,\left|P_{2}^{-}\right\rangle$as well as the product states exactly in the valence bond (VB) basis ${ }^{28}$ (in which the total spin $S$ is a good quantum number) in order to avoid spin contamination. Following the time evolution, however, we need to calculate overlaps of the time-evolved states with various final states (see below), which is cumbersome within the nonorthogonal VB basis. After calculating the exact spin singlet and triplet initial states, we therefore expand these in an orthonormal basis that has only well defined total $M_{S}$ value.

Henceforth we refer to the initial states $\left|i_{S}\right\rangle$ and $\left|i_{T}\right\rangle$ collectively as $\Psi(0)$ and the time-evolved states as $\Psi(t)$. In principle, the time evolution can be done by operating on $\Psi(0)$ with the time evolution operator

$$
U(0, t)=\exp (-i H t),
$$

where $H$ is the total Hamiltonian $H_{\text {intra }}+H_{\text {inter }}$. This approach would, however, require obtaining a matrix representation of the exponential time-evolution operator, which in turn requires the determination of the prohibitively large number of eigenstates of the composite two-chain system. We can avoid this problem by using small discrete time intervals and expanding the exponential operator in a Taylor series, and stopping at the linear term. Such an approach, however, has the undesirable effect of spoiling unitarity, and for long time evolutions would lead to loss of normalization of the evolved state. The way around this dilemma has been proposed and used by others ${ }^{33,34}$ in different contexts and involves using the following truncated time-evolution scheme,

$$
\left(1+i H \frac{\Delta t}{2}\right) \Psi(t+\Delta t)=\left(1-i H \frac{\Delta t}{2}\right) \Psi(t) .
$$

In the above equation, on the left-hand side, we evolve the state at time $(t+\Delta t)$ backwards by $\Delta t / 2$, while on the righthand side we evolve the state at time $t$ forward by $\Delta t / 2$. By forcing these two to be equal, we ensure unitarity in the time evolution of the state. It can be seen easily that this time evolution which is accurate to $\Delta t^{2} / 2$ is unitary. For a given many-body Hamiltonian and initial state, the right-hand side of Eq. (10) is a vector in the Hilbert space of the two-chain Hamiltonian. The left-hand side corresponds to the action of a matrix on an as yet unknown vector, that is obtained by 
solving the above set of linear algebraic equations. Further details of the numerical procedure can be found in Appendix B.

After each evolution step, the evolved state is projected onto the space of neutral product eigenstates of the two-chain system. The relative yield $I_{m n}(t)$ for a given product state $|m, n\rangle=|m\rangle_{1}|n\rangle_{2}$ is then obtained from

$$
I_{m n}(t)=|\langle\Psi(t) \mid m, n\rangle|^{2} .
$$

In our case the states $|m, n\rangle$ can be any of the final states of interest, viz., $\left|\left(1{ }^{1} A_{g}\right)_{1}\left(1^{1} B_{u}\right)_{2}\right\rangle,\left|\left(1^{1} A_{g}\right)_{1}\left(1^{3} B_{u}\right)_{2}\right\rangle$, etc. It is for efficient calculations of the overlaps (while at the same time maintaining spin purity) in Eq. (11) that we expand our exact eigenstates of the neutral system in the VB basis to the total $M_{S}$ basis. We emphasize that $I_{m n}(t)$ is a measure of the yield of the state $|m, n\rangle$ at time $t$ and is not a cross section.

\section{NUMERICAL RESULTS}

In this section we report the results of our calculations of recombination dynamics for for pairs of ethylenes, butadienes, and hexatrienes, both within the noninteracting Hückel model $\left(U_{i}=V_{i j}=X_{\perp}=0\right)$ and the interacting PPP model. Following this, we show the results of our investigation of electric field effects on the same systems, discuss the chain length dependence of $\eta$, and finally present the numerical results for a model system containing nitrogen heteroatoms. The calculations for the noninteracting case provides a check of our numerical procedure, and the comparison between the noninteracting and the interacting model allows us to determine the effect of electron-electron interactions.

\section{A. Dynamics in the Hückel Model}

While there is no difference in energy between singlets and triplets in the Hückel model, it is nevertheless possible to have spin singlet and triplet initial states $\left|i_{S}\right\rangle$ and $\left|i_{T}\right\rangle$, as well as singlet and triplet final states. In Fig. 2 we show the yield for the electron-hole recombination in the singlet channel, and for pairs of ethylenes, butadienes and hexatrienes. The yields for the triplet channels are not shown separately in this case; we have ascertained that these are identical to those in the singlet channel in this case, as expected. These calculations are for $t_{\perp}=0.1 \mathrm{eV}$ within Eq. (6). We note that the yields $I_{m n}(t)$ oscillate with time. This is to be expected within our purely electronic Hamiltonian, within which an electron or hole jumps back and forth between the two molecular species. These oscillations are the analogs of the Rabi oscillations $^{35,36}$ that occur upon the stimulation of a system with light, where absorption of light can occur only with nonzero damping. Within our purely electronic Hamiltonian, complete transition to the final states can only occur in the presence of damping (for example, radiative and nonradiative relaxations of the final states), which has not been explicitly included in our Hamiltonian. The frequency of oscillation is higher for larger intermolecular transfer integral $t_{\perp}$, as expected. The frequency of the oscillation also depends
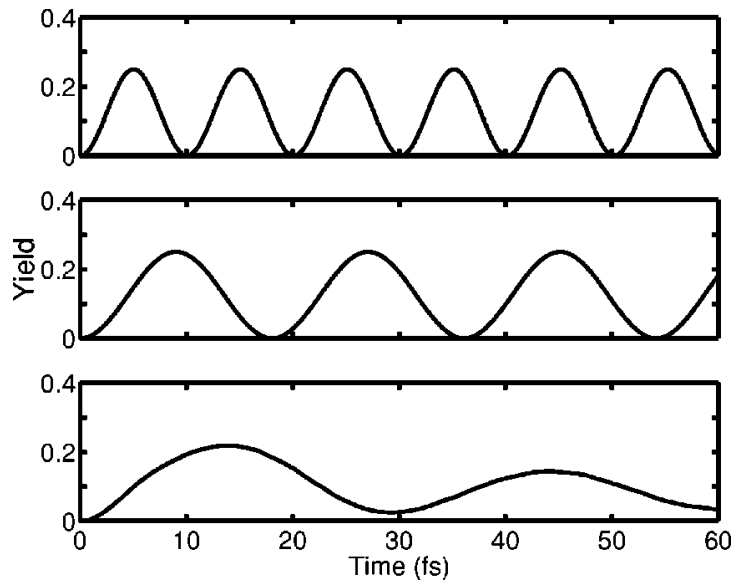

FIG. 2. Yield in the singlet channel as a function of time, for pairs of ethylenes (top panel), butadienes (middle panel), and hexatrienes (bottom panel), within the simple Hückel model ( $U$ $\left.=V_{i j}=X_{\perp}=0\right)$. Significant yield in all cases occur only for final states $\left|\left(1^{1} A_{g}^{+}\right)_{1}\left(1^{1} B_{u}^{-}\right)_{2}\right\rangle$ and $\left|\left(1^{1} B_{u}^{-}\right)_{1}\left(1^{1} A_{g}^{+}\right)_{2}\right\rangle$, between which the yields are identical. Yields in the triplet channel $\left|\left(1{ }^{1} A_{g}^{+}\right)_{1}\left(1{ }^{3} B_{u}^{+}\right)_{2}\right\rangle$ and $\left|\left(1{ }^{3} B_{u}^{+}\right)_{1}\left(1{ }^{1} A_{g}^{+}\right)_{2}\right\rangle$ are identical to those in the singlet channel. Calculation of the full period of oscillation for hexatrienes would take much longer time evolution than $60 \mathrm{fs}$ and is not shown.

upon the size of the molecule and is lower for larger molecules (see below for an explanation of this). The equalities in the yields of the singlet and triplet excited states found numerically conforms to the simple free-spin statistics which predicts that in the $M_{S}=0$ state formed from electron-hole recombination, the probability of singlet and triplet formation are equal. Since the $M_{S}= \pm 1$ cases always yield triplets, the spin statistics corresponding to $25 \%$ singlets and $75 \%$ triplets is vindicated in this case.

Although the Hückel calculations do not yield any new information, it is useful to pursue them further in order to arrive at a physical mechanism of the charge recombination process. To this end we have developed an alternate procedure for calculating the above dynamics for the smallest model system, viz., a pair of ethylenes. This alternate approach consists of expanding the initial state $\Psi(0)$ as a superposition of the eigenstates $\psi_{i}$ of the composite two-chain system with eigenvalues $E_{i}$,

$$
|\Psi(0)\rangle=\sum_{i} c_{i}\left|\psi_{i}(0)\right\rangle
$$

The evolution of the state $\Psi(0)$ is now simply given by

$$
|\Psi(t)\rangle=\sum_{i} c_{i}\left|\psi_{i}(0)\right\rangle \exp \left(-i E_{i} t / \hbar\right)
$$

The yield $I_{m n}(t)$ in a given channel with final state $|m, n\rangle$ is then obtained from 
TABLE I. Significant $c_{i}=\left\langle\Psi(0) \mid \psi_{i}\right\rangle$ and the $\left\langle m, n \mid \psi_{i}\right\rangle$ values and their product in the Hückel model for a pair ethylenes in singlet channel. The index $i$ corresponds to the index of "significant" eigenstates of the total system and $E_{i}$ the corresponding energy eigenvalue.

\begin{tabular}{lcccc}
\hline \hline$i$ & $E_{i}(\mathrm{eV})$ & $c_{i}$ & $\left\langle m, n \mid \psi_{i}\right\rangle$ & $\left\langle m, n \mid \psi_{i}\right\rangle c_{i}$ \\
\hline 2 & -4.3360 & 0.3691 & 0.1362 & 0.0503 \\
3 & -4.3360 & -0.3373 & 0.1138 & -0.0384 \\
4 & -4.1360 & -0.0171 & 0.0120 & -0.0002 \\
5 & -4.1360 & -0.5000 & 0.0058 & -0.0029 \\
6 & -4.1360 & 0.4989 & 0.0120 & 0.0057 \\
7 & -4.1360 & -0.0285 & 0.0059 & -0.0002 \\
8 & -3.9360 & -0.3558 & 0.1266 & -0.0450 \\
9 & -3.9360 & 0.3513 & 0.1234 & 0.0433 \\
\hline \hline
\end{tabular}

$$
\begin{aligned}
I_{m n}(t)= & |\langle m, n \mid \Psi(t)\rangle|^{2}=\sum_{i}\left|c_{i}\left\langle m, n \mid \psi_{i}(0)\right\rangle \exp \left(-i E_{i} t / \hbar\right)\right|^{2} \\
= & \sum_{i}\left|c_{i}\left\langle m, n \mid \psi_{i}(0)\right\rangle\right|^{2} \\
& +\sum_{i} \sum_{j>i} 2 \operatorname{Re}\left\{c_{i} c_{j}\left\langle m, n \mid \psi_{i}(0)\right\rangle\left\langle\psi_{j}(0) \mid m, n\right\rangle\right\} \\
& \times \cos \left[\left(E_{i}-E_{j}\right) t / \hbar\right] .
\end{aligned}
$$

The quantities $\left\langle m, n \mid \psi_{i}(0)\right\rangle$ are readily obtained from the eigenstates of the neutral one-chain subsystems and the composite eigenstates of the two-chain system. In Tables I and II we list the nonzero values of the coefficients $c_{i}$ and the $\left\langle m, n \mid \psi_{i}(0)\right\rangle$ values for the case of two ethylenes. It is seen that sets of degenerate states of the composite system together contribute equally to the singlet and triplet channels, although individual members of the set may contribute unequally. We have determined that the time evolution obtained from this approach is exactly the same as that obtained from the general method described in the previous section.

The contribution arising from the right-hand side of Eq. (14) has been separated into time-independent and timedependent parts. The latter comes about whenever the two eigenstates in question are nondegenerate. Furthermore, at $t$ $=0$ the contribution from the time-independent part exactly

TABLE II. Significant $c_{i}=\left\langle\Psi(0) \mid \psi_{i}\right\rangle$ and $\left\langle m, n \mid \psi_{i}\right\rangle$, for the triplet channel, for a pair of ethylenes.

\begin{tabular}{lcccc}
\hline \hline$i$ & $E_{i}(\mathrm{eV})$ & $\left\langle\Psi(0) \mid \psi_{i}\right\rangle$ & $\left\langle m, n \mid \psi_{i}\right\rangle$ & $\left\langle m, n \mid \Psi_{i}\right\rangle c_{i}$ \\
\hline 2 & -4.3360 & 0.3373 & 0.1138 & 0.0384 \\
3 & -4.3360 & 0.3691 & 0.1362 & 0.0503 \\
4 & -4.1360 & -0.0179 & 0.0037 & -0.0001 \\
5 & -4.1360 & 0.4985 & 0.0180 & 0.0090 \\
6 & -4.1360 & 0.5005 & 0.0047 & 0.0024 \\
7 & -4.1360 & 0.0251 & 0.0170 & 0.0004 \\
8 & -3.9360 & 0.3513 & 0.1234 & 0.0433 \\
9 & -3.9360 & 0.3558 & 0.1266 & 0.0450 \\
\hline \hline
\end{tabular}

cancels the contribution from the time-dependent part. When the sign of the time-dependent part becomes positive, the two contributions add up to give the maximum yield of 0.25 in both the singlet and the triplet channels observed in the discrete calculations. The periodicity of the oscillation corresponds to the energy difference between the two pairs of the degenerate states. This analysis could in principle be extended to the case of the larger systems but would be quite tedious in view of the larger Hilbert space dimensions. Note that the decrease of the oscillation frequency of $I_{m n}(t)$ with increasing chain length (Fig. 2) is explained within the above alternate procedure. The length dependence of the oscillation frequency originates from the smaller $E_{i}-E_{j}$ in longer chains.

\section{B. Dynamics in the PPP model}

We now present our results for interacting electrons in $H_{\text {intra }}$ and $H_{\text {inter }}$. In all cases for the interchain $V_{i, j}$ we have chosen the Ohno parameters, and the interchain hopping $t_{\perp}$ $=0.1 \mathrm{eV}$. For $X_{\perp}$, we present the results of calculations with both $X_{\perp}=0$ and $0.1 \mathrm{eV}$. In Figs. 3(a) and 3(b) we show the plots of $I_{m n}(t)$ in the singlet and triplet channels for pairs of ethylenes, butadienes and hexatrienes, respectively, for the case of $X_{\perp}=0$. The same results are shown in Figs. 3(c) and 3(d) for $X_{\perp}=0.1 \mathrm{eV}$.

The most obvious difference from the Hückel model is that the yields $I_{m n}(t)$ in both the singlet and triplet channels are considerably reduced in the present cases. Two other points are to be noted. First, there is now substantial difference between the singlet and triplet channels, with the singlet yield higher in all cases. Second, the strong differences in singlet and triplet yields are true for both $X_{\perp}=0$ and $X_{\perp} \neq 0$. This is in contradiction to the golden rule approach, ${ }^{15,16}$ which ignores the energy difference between the $1^{1} B_{u}$ and the $1^{3} B_{u}$. The only consequence of nonzero $X_{\perp}$ is the asymmetry between the yields of $\left(1{ }^{1} A_{g}\right)_{1}\left(1{ }^{1} B_{u}\right)_{2}$ and $\left(1{ }^{1} A_{g}\right)_{2}\left(1{ }^{1} B_{u}\right)_{1}$ in the singlet channels, and a similar asymmetry in the triplet channels. Further discussion of this asymmetry can be found in Appendix A. The overall conclusion that emerges from the results of Figs. 3(a)-3(d) is that nonzero electron-electron interactions substantially enhances $\eta$.

We point out that the oscillatory nature of $I_{m n}(t)$ in the absence of damping is real, but it does not imply a timedependent $\eta$. In the absence of damping, the yield of a given state can in principle be obtained by an integration of $I_{m n}(t)$ over one complete period, and $\eta$ would simply be the ratio of two such integrated yields. We have not attempted such integration because of the following reasons. Firstly, our goal is to obtain qualitative information only, and the plots of $I_{m n}(t)$ for the singlet and triplet yields are so strongly different for nonzero Coulomb interactions that the enhancement of $\eta$ is obvious in all cases. Second, and more importantly, as seen in Figs. 3(c) and 3(d), nonzero $X_{\perp}$ creates a substantial asymmetry in the yields, and this information would be lost upon integration. On the other hand, this information is important, from the viewpoints of our discussions of the role of $X_{\perp}$ in Sec. III and here, and the possibility of confirming 

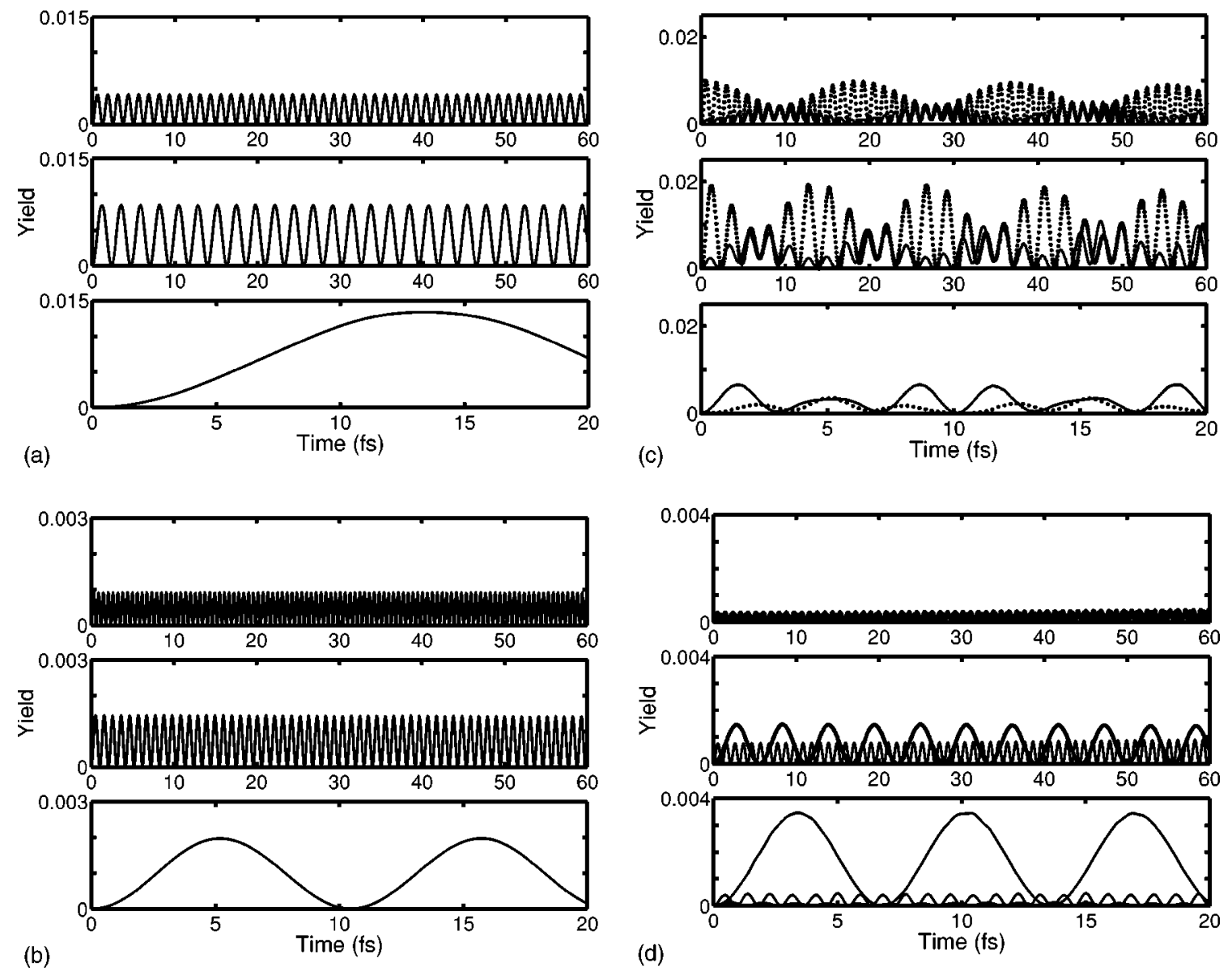

FIG. 3. Yields in the singlet and triplet channels within the PPP Hamiltonian. In all cases the top panel corresponds to pair of ethylenes, the middle panel to pairs of butadienes, and the bottom panel to pairs of hexatrienes. (a) Singlet channel, $t_{\perp}=0.1 \mathrm{eV}, X_{\perp}=0 ;(\mathrm{b})$ triplet channel, $t_{\perp}=0.1 \mathrm{eV}, X_{\perp}=0$; (c) singlet channel, $t_{\perp}=0.1 \mathrm{eV}, X_{\perp}=0.1 \mathrm{eV}$; (d) triplet channel $t_{\perp}=0.1 \mathrm{eV}, X_{\perp}=0.1 \mathrm{eV}$. Evolution in the case of hexatrienes is tracked for $20 \mathrm{fs}$ while in other cases, the evolution is tracked for $60 \mathrm{fs}$. Calculation of the full period of oscillation for hexatrienes would take much longer time than $20 \mathrm{fs}$ and is not shown. Significant yields in singlet channel occurs only for final states $\left|\left(1{ }^{1} A_{g}^{+}\right)_{1}\left(1^{1} B_{u}^{-}\right)_{2}\right\rangle$ and $\left|\left(1{ }^{1} B_{u}^{-}\right)_{1}\left(1^{1} A_{g}^{+}\right)_{2}\right\rangle$, between which the yields are identical in (a) and (b) but different in (c) and (d). Similarly, yields in triplet channel are to the states $\left|\left(1^{1} A_{g}^{+}\right)_{1}\left(1^{3} B_{u}^{+}\right)_{2}\right\rangle$ and $\left|\left(1^{3} B_{u}^{+}\right)_{1}\left(1^{1} A_{g}^{+}\right)_{2}\right\rangle$, between which the yields are identical in (a) and (b) but different in (c) and (d).

such asymmetries experimentally in the future. Third, in time-dependent calculations within the sudden approximation, where the perturbation is switched on abruptly, there is often a spurious oscillatory behavior at the earliest times when energy conservation may be violated. Such spurious oscillations decay very rapidly, and the continuation of the oscillations in our plots with the same periodicities and amplitudes indicates that the behavior shown is real. Finally, quantitative estimate of $\eta$ can only be done by including the damping mechanisms within a rate equation formalism, thus rendering the integration of the curves shown pointless in any case. What is important is to realize though that inclusion of the damping terms will not change our conclusion that $\eta>0.25$. This is because the lifetimes of both the singlet and triplet excitons are larger than the periods of oscillation, and the lifetime of the triplet exciton is considerably larger than that of the singlet exciton. While the oscillation is going on in the singlet channel, if the exciton decays to the ground state additional singlet excitons will form from general ki- netic theory principles. Since such decays occur less frequently in the triplet channel, this further enhances $\eta$.

In order to understand the above results in further detail we have also carried out the dynamics calculation for pairs of ethylenes according to Eq. (14). As in the Hückel case these calculations yield the same results as the more general method. Our results for the wave functions of the composite two-chain system and the overlaps of the product eigenstates of the final neutral molecules with these are shown in Tables III and IV. The degeneracies in the eigenstates of the composite system that characterized the Hückel model are now lifted, which is a known electron correlation effect. What is more significant in the present case is that the composite state wave functions that have large overlaps with $\Psi(0)$ are now not the same ones that have large overlaps with the product wave functions of the final states. This is what reduces the yields of the charge-transfer processes in the PPP model, relative to the Hückel model.

Tables III and IV give a clear physical picture of the charge recombination process. For a large yield what appears 
TABLE III. Significant $c_{i}=\left\langle\Psi(0) \mid \psi_{i}\right\rangle$ and the $\left\langle m, n \mid \psi_{i}\right\rangle$ values and their product for PPP model in the absence of electric field, for a pair of ethylenes in the singlet channel. The index $i$ corresponds to the index of 'significant' eigenstates of the total system and $E_{i}$ the corresponding energy eigenvalue.

\begin{tabular}{lcccc}
\hline \hline & $E_{i}$ & $c_{i}$ & $\left\langle m, n \mid \psi_{i}\right\rangle$ & $c_{i}\left\langle m, n \mid \psi_{i}\right\rangle$ \\
\hline 4 & 0.5295 & -0.0249 & -0.6992 & 0.0174 \\
5 & 0.7328 & -0.0458 & -0.6953 & 0.0318 \\
11 & 3.7748 & 0.7066 & -0.0258 & -0.0182 \\
13 & 3.7844 & -0.7056 & 0.0446 & -0.0315 \\
29 & 11.2503 & 0.0082 & 0.1020 & 0.0008 \\
30 & 11.6379 & -0.0025 & 0.1206 & -0.0003 \\
32 & 14.0483 & 0.0050 & 0.0028 & 0.00001 \\
34 & 14.0611 & -0.0054 & 0.0081 & -0.00004 \\
\hline \hline
\end{tabular}

to be essential is that the composite two-chain system must have at least some eigenstates which have simultaneously large overlaps with both the direct product of the initial polaronic states and the direct product of the pair of eigenstates of the neutral subsystems in the the chosen channel. This can be interpreted as a "transition state theory" for the charge recombination reaction of Eq. (1). Large overlaps with the initial polaronic pair states occur for the states 11 and 13 in the singlet channel (see Table III), and for the states 10 and 12 in the triplet channel (see Table IV). This is in contrast to the Hückel case, where the large overlaps with the polaron pair wave functions were with the same composite two-chain eigenstates. The overlaps of these specific two chain eigenstates are larger for products of singlet final states $\left|1{ }^{1} A_{g}\right\rangle_{1}\left|1^{1} B_{u}\right\rangle_{2}$ than for triplet final states $\left|1^{1} A_{g}\right\rangle_{1}\left|1{ }^{3} B_{u}\right\rangle_{2}$, and this is what gives a larger yield for the singlet exciton.

\section{Effects of external electric field}

Our results in the previous subsection already indicate that $\eta$ can be substantially larger than 0.25 for the correlated electron Hamiltonian of Eq. (4). From comparison of Fig. 2 and Figs. 3(a)-3(d), we see however, that the relative yields $I_{m n}(t)$ are lower by orders of magnitude for interacting electrons. This is easily understandable within time-independent

TABLE IV. Significant $c_{i}=\left\langle\Psi(0) \mid \psi_{i}\right\rangle$ and the $\left\langle m, n \mid \psi_{i}\right\rangle$ values and their product for PPP model in the absence of electric field, for a pair of ethylenes in the triplet channel.

\begin{tabular}{lcccc}
\hline \hline & $E_{i}$ & $c_{i}$ & $\left\langle m, n \mid \psi_{i}\right\rangle$ & $c_{i}\left\langle m, n \mid \psi_{i}\right\rangle$ \\
\hline 2 & -2.7283 & -0.0215 & -0.6980 & 0.0150 \\
3 & -2.7238 & -0.0091 & 0.6982 & -0.0064 \\
10 & 3.7697 & 0.7068 & 0.0060 & 0.0042 \\
12 & 3.7775 & -0.7067 & 0.0203 & -0.0143 \\
19 & 8.0804 & -0.0056 & 0.1115 & -0.0006 \\
20 & 8.0875 & -0.0190 & -0.1114 & 0.0021 \\
31 & 14.0475 & 0.0051 & -0.0056 & -0.00003 \\
33 & 14.0515 & -0.0052 & -0.0023 & 0.00001 \\
\hline \hline
\end{tabular}
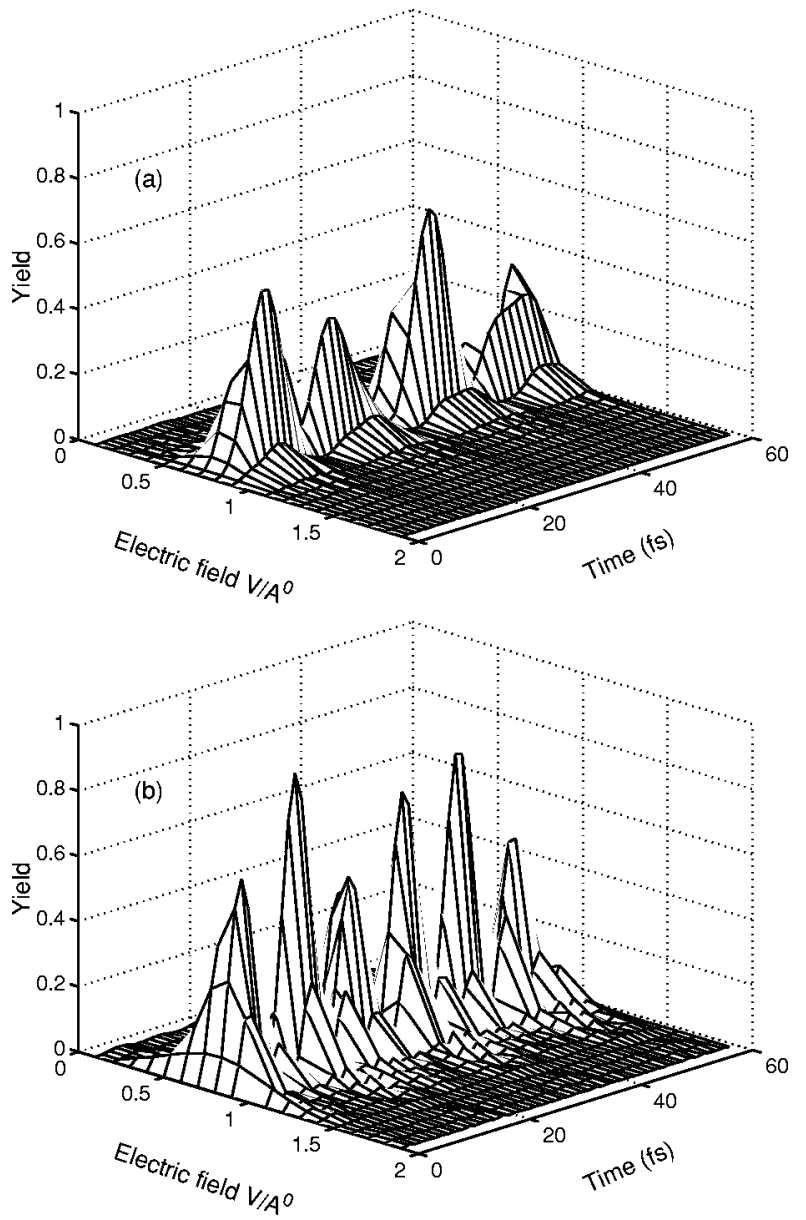

FIG. 4. Yields in the singlet channels (a) $\left|\left(1{ }^{1} A_{g}\right)_{1}\left(1^{1} B_{u}\right)_{2}\right\rangle$, (b) $\left|\left(1{ }^{1} A_{g}\right)_{2}\left(1{ }^{1} B_{u}\right)_{1}\right\rangle$, as a function of the electric field $(\mathrm{V} / \AA)$ and time (fs). Here $t_{\perp}=0.1 \mathrm{eV}$ and $X_{\perp}=0.1 \mathrm{eV}$.

second order perturbation theory, within which the extent to which the initial polaron-pair state is modified is directly proportional to the matrix element of $H_{\text {inter }}$ between the initial and final states, and inversely proportional to the zeroth order energy difference. Since the energy differences between the polaron-pair states and the final neutral states are substantial within the PPP Hamiltonian, the yields are low. There are two possible interpretations of these results. First, the actual yields of excitons in OLED's is indeed low, compared to the theoretical maximum for noninteracting electrons (recall that no direct comparison of the experimental light emission intensities with the theoretical maximum is possible). Second, the experimentally observed yields are influenced substantially by external factors ignored so far. We consider this second possibility here, and calculate within our time-dependent formalism the yields $I_{m n}(t)$ in the presence of an external electric field ("external" in the following includes the effects of both the actual bias voltage as well as all internal field effects). What follows may be thought of as overly simple, but nevertheless, we believe that it gives the correct physical picture. We first present our formalism and numerical calculations, and only then we discuss the interpretation of these results. 

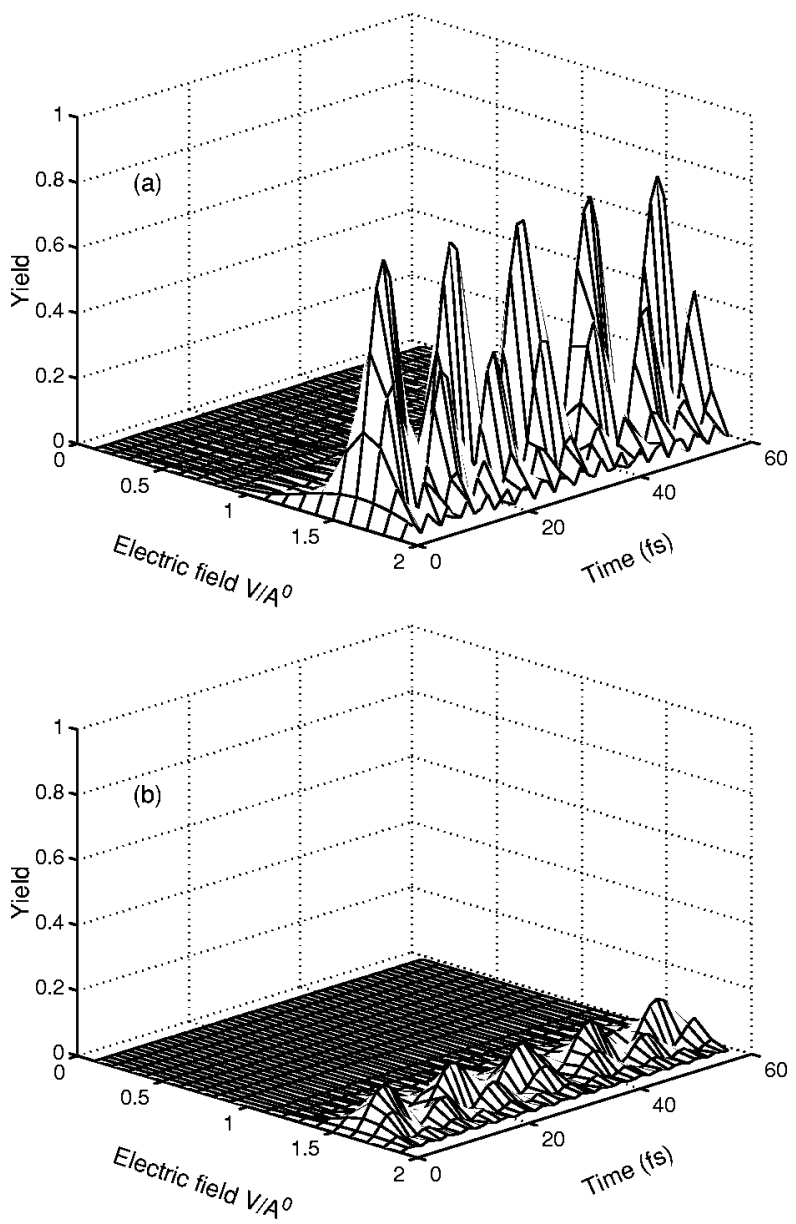

FIG. 5. Yields in the triplet channels (a) $\left|\left(1{ }^{1} A_{g}\right)_{1}\left(1^{3} B_{u}\right)_{2}\right\rangle$, (b) $\left|\left(1^{1} A_{g}\right)_{2}\left(1^{3} B_{u}\right)_{1}\right\rangle$, as a function of the electric field $(\mathrm{V} / \AA)$ and time (fs). Parameters are same as in Fig. 4.

As before, we consider pairs of molecules that are parallel to each other, with the molecular chain axes aligned parallel to the $x$ axis. The electric field is chosen along the $y$ axis, such that the total Hamiltonian now has an additional contribution,

$$
H_{\text {field }}=E \sum_{i}\left[\left(n_{i}-1\right) y_{i}+\left(n_{i}^{\prime}-1\right) y_{i}^{\prime}\right]
$$

In the above $E$ is the strength of the electric field, and $y_{i}\left(y_{i}^{\prime}\right)$ gives the $y$ component of the location of the $i$ th $\left(i^{\prime}\right.$ th carbon atom in molecule 1 (2). We now perform our dynamical calculations with the complete Hamiltonian including $H_{\text {field }}$. Notice that there occurs no change in the initial states in the presence of the electric field, since the electric field is taken to be perpendicular to the linear molecules. This is of course a theoretical ideal chosen for computational convenience (as is the model of two exactly parallel chains), and in real systems the orientations of the field and the molecules are random (see below for further discussions).

In Figs. 4 and 5 we show the effect of the external electric field on the yield in the singlet and triplet channels for a pair of ethylenes. We see that in all the cases there is a strong nonlinear dependence of the yield on the external field. In
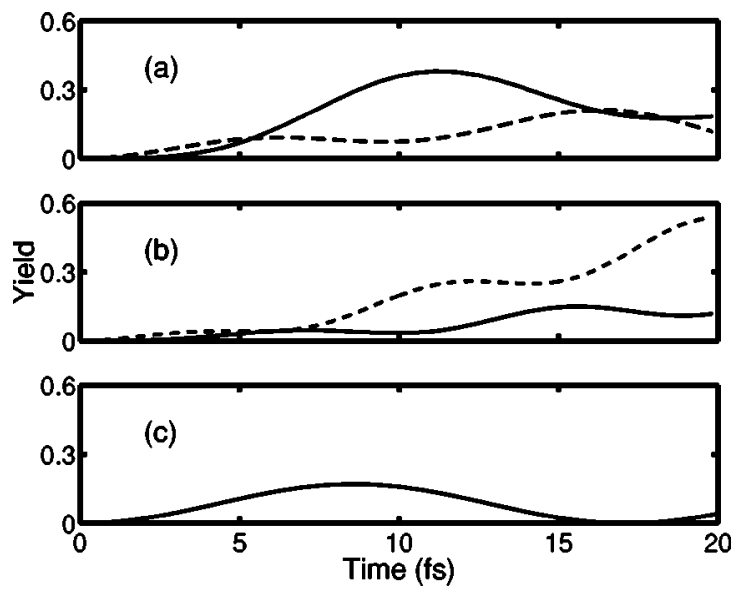

FIG. 6. Yields in the singlet channel for pairs of hexatriene molecules, as a function of time (fs) with $t_{\perp}=0.1 \mathrm{eV}$ and $X_{\perp}$ $=0.1 \mathrm{eV}$ in an external electric field. (a) Singlet channel at $0.3 \mathrm{~V} / \AA$, (b) singlet channel at $0.42 \mathrm{~V} / \AA$ and triplet channel at $1.0 \mathrm{~V} / \AA ̊$.

both the singlet and the triplet channels, we see sharp increases in the yields over a range of field strengths. The field strengths at which the increases in the yields occur are about two orders of magnitude larger than the experimental fields in the OLED's, and we comment upon this below. Here we only observe that the field strength $E$ over which the singlet yield is larger is smaller than the field strength over which the triplet yield dominates.

We have performed similar calculations for the longer chain systems, and in all cases the effects are the same, viz., there exists a range of field strength where a sudden increase in the singlet yield occurs, while at still larger fields there occurs a similar jump in the triplet yield. In Figs. 6(a) and 6(b) we have shown the singlet and triplet yields for field strengths of $0.3 \mathrm{~V} / \AA$ and $1.0 \mathrm{~V} / \AA$, respectively, for hexatriene. In general, for a given spin channel the threshold field strength decreases with the chain length (the threshold field for the singlet channel decreases from $0.7 \mathrm{~V} / \AA$ to $0.3 \mathrm{~V} / \AA$ on going from ethylene to hexatriene, while the threshold field for the triplet channel decreases from $1.6 \mathrm{~V} / \AA$ to $1.0 \mathrm{~V} / \AA)$. The most important conclusions that emerge from these calculations are that, (a) macroscopically observable yields, comparable to the zero-field yields within the noninteracting Hückel model, are found for large fields, and (b) while the calculated $\eta$ are greater than 0.25 for smaller fields, this is reversed with further increase in the field strength.

In order to understand the origin of the increased yields over ranges of the electric field, we have analyzed the case of a pair of ethylenes extensively, within Eq. (14). Firstly it is worth noting that the geometry in which the field is introduced, the product states of the neutral Hamiltonian are unaffected by the electric field. We also notice that the eigenvalues of the total Hamiltonian are not very sensitive to the external field. As in the field-free cases, we have obtained the projections of the eigenstates of the two-chain system on the initial state as well as the product of the final states, as a function of the applied electric field in both the singlet and 

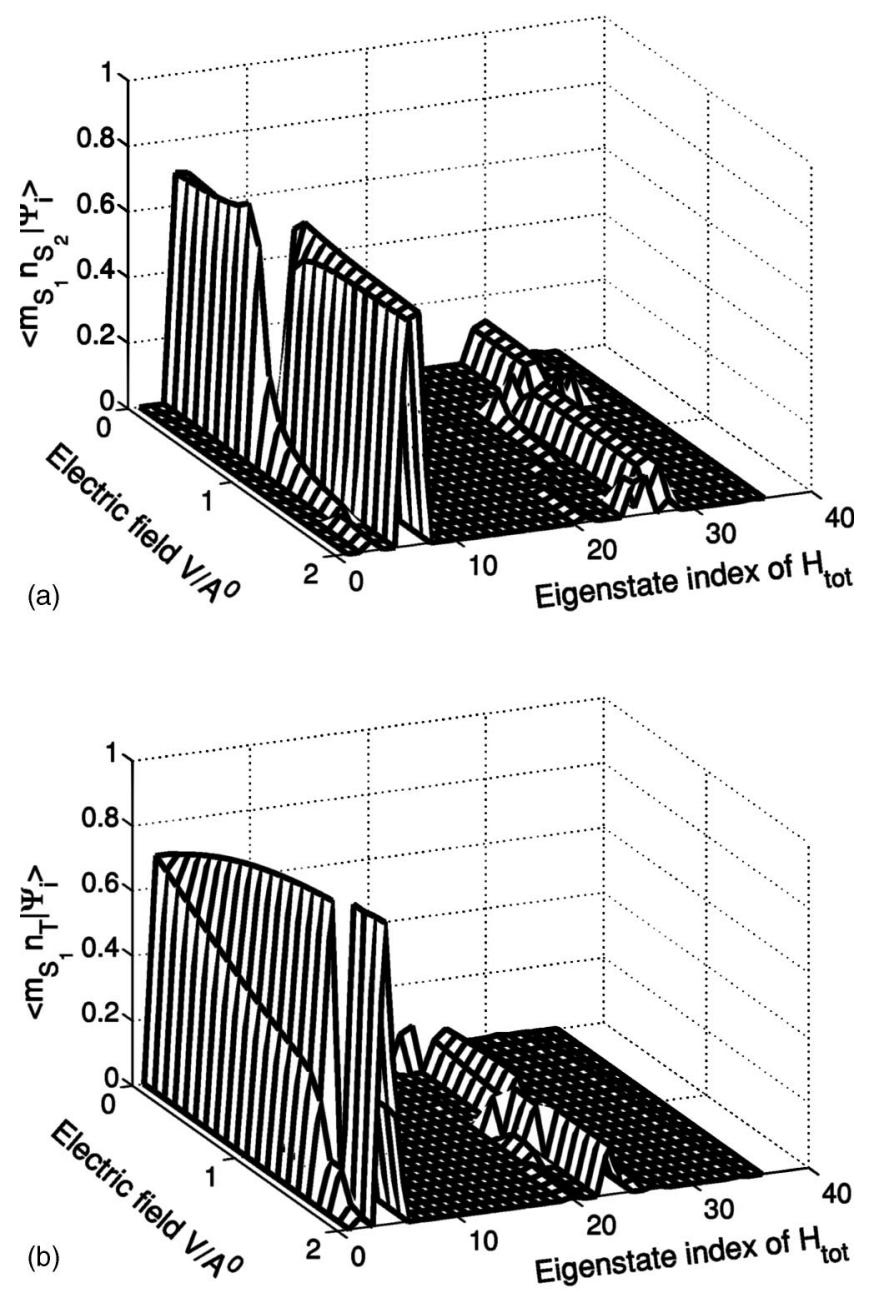

FIG. 7. Evolution of significant $\left\langle\psi_{i} \mid m, n\right\rangle$ as a function of electric field (V/Å), in the case of the explicit time evolution of eigenvectors the PPP Hamiltonian for a pair of ethylenes in (a) singlet and (b) triplet channels.

the triplet channels. In Fig. 7, we plot the coefficients $\left\langle\psi_{i} \mid m, n\right\rangle$ as a function of the electric field for the singlet and the triplet channels. We see that there are several states that show strong variation in both cases as a function of the field. However, when a product of these coefficients with $\left\langle\Psi(0) \mid \psi_{i}\right\rangle$ is analyzed, the number of the states that simultaneously have a large value of these coefficients at the same electric field is smaller. In Fig. 8, we plot the dominant coefficients of these projections, as a function of the applied field. We note that only a few states have both projections simultaneously large. We also note that both the projections peak at the same field strength. It is this that leads to an abrupt increase in the yield at that field strength. The eigenstates of the full Hamiltonian that have large projections simultaneously to both the initial and the final states can in fact be expressed almost completely as a linear combination of the initial polaron product state and the final product state of the neutral system eigenstates. In Tables V and VI, we show the projections of the eigenstates of the full Hamiltonian at the resonant electric field on to (i) the initial state and (ii) to the product of eigenstates of the neutral system for which
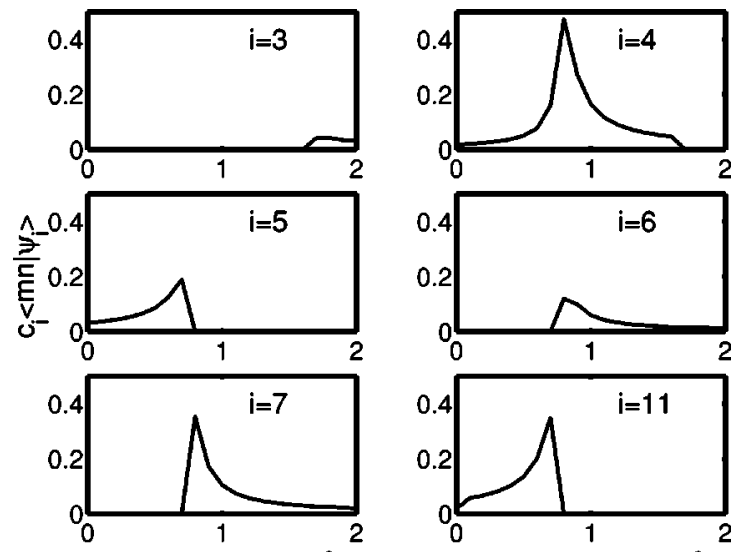

(a)
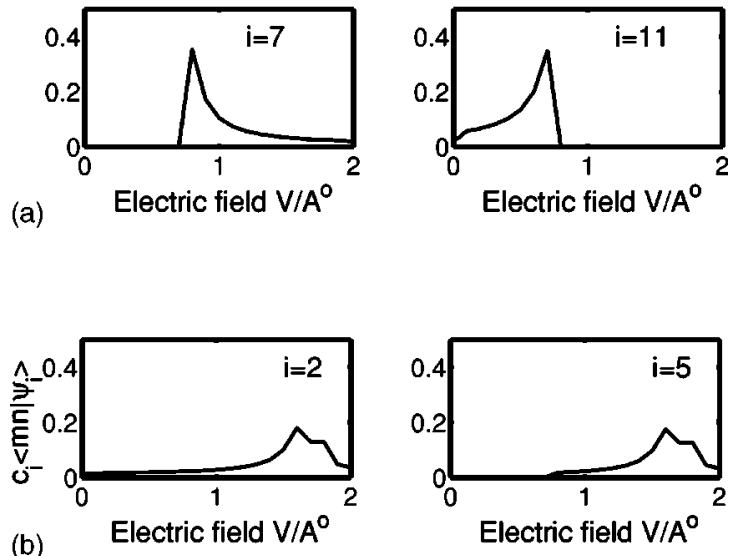

FIG. 8. $\left\langle m, n \mid \psi_{i}\right\rangle\left\langle\Psi(0) \mid \psi_{i}\right\rangle$ plotted as a function of electric field $(\mathrm{V} / \AA)$ ), for significant states $i$ for (a) the singlet-singlet channel and (b) the singlet-triplet channel for a pair of ethylenes. The singlet-singlet channel in (a) corresponds to $\left|m_{S_{1}}\right\rangle$ and $\left|n_{S_{2}}\right\rangle$ and the singlet-triplet channel in (b) corresponds to $\left|m_{S_{1}}\right\rangle$ and $\left|n_{T}\right\rangle$.

resonance is observed. We note that there are a few eigenstates of the full Hamiltonian which have large coefficients for both projections. This seems to be independent of the energy of the eigenstate of the total system. The energetics decide the period of oscillations and not the amplitude of the oscillations.

We now come to our interpretations of the above numerical calculations. In all cases the applied fields in our calculations are substantially larger than what is expected from the externally applied voltage in OLED's. Note, however, that our molecules are rather small, and the calculated threshold fields at which the effect becomes observable decrease with the molecular size. In this context, it is worth recalling a previous exact calculation of electroabsorption for short finite polyenes. ${ }^{37}$ There the electric field was parallel to the chain axis (as opposed to perpendicular, as in the present

TABLE V. In the case of a pair of ethylenes the states with significant $c_{i}=\left\langle\Psi(0) \mid \psi_{i}\right\rangle$ and $\left\langle m, n \mid \psi_{i}\right\rangle$, for the PPP model with electric field of $0.7 \mathrm{~V} / \AA$ in the singlet channel.

\begin{tabular}{lcccc}
\hline \hline$i$ & $E_{i}(\mathrm{eV})$ & $c_{i}$ & $\left\langle m, n \mid \psi_{i}\right\rangle$ & $c_{i}\left\langle m, n \mid \psi_{i}\right\rangle$ \\
\hline 4 & 0.5153 & 0.2086 & 0.7629 & 0.1591 \\
5 & 0.6828 & 0.3824 & 0.4933 & 0.1886 \\
11 & 1.0479 & -0.9001 & 0.3868 & -0.3482 \\
21 & 6.5753 & 0.0005 & -0.0055 & 0.0000 \\
27 & 11.2881 & -0.0016 & -0.0577 & 0.0001 \\
30 & 11.6946 & 0.0040 & -0.1175 & -0.0005 \\
\hline \hline
\end{tabular}


TABLE VI. In the case of a pair of ethylenes the states with significant $c_{i}=\left\langle\Psi(0) \mid \psi_{i}\right\rangle$ and $\left\langle m, n \mid \psi_{i}\right\rangle$, for the PPP model with electric field of $1.6 \mathrm{~V} / \AA$ in the triplet channel.

\begin{tabular}{lcccc}
\hline \hline$i$ & $E_{i}(\mathrm{eV})$ & $c_{i}$ & $\left\langle m, n \mid \psi_{i}\right\rangle$ & $c_{i}\left\langle m, n \mid \psi_{i}\right\rangle$ \\
\hline 2 & -2.8347 & 0.5927 & 0.3031 & 0.1796 \\
3 & -2.7237 & 0.0052 & 0.9142 & 0.0048 \\
5 & -2.5174 & 0.8053 & -0.2179 & -0.1755 \\
20 & 7.5928 & 0.0089 & -0.0180 & -0.0002 \\
23 & 8.0821 & 0.0054 & 0.1518 & 0.0008 \\
24 & 8.1387 & -0.0031 & 0.0364 & -0.0001 \\
26 & 10.1769 & 0.0001 & 0.0163 & 0.0000 \\
\hline \hline
\end{tabular}

case), and it was found that the calculated electroabsorption can simulate the experimentally observed behavior in longchain polymers, ${ }^{38,39}$ provided the electric field used in the short chain calculation was larger by two orders of magnitude than the experimental field. This is because of the large energy gaps in short chains. We believe that a similar argument applies in the present calculations of interchain charge transfer: the energy difference between the initial polaronpair state and the final states is much larger in the small molecule-pair system than in the experimental systems, even when oligomeric. The analogy to electroabsorption would then imply that the enhanced macroscopic yields would occur in the real systems at much smaller, perhaps even realistic fields.

One final point concerns the geometry used in our calculations. In real OLED's the relative orientations of the molecules of a given pair, as well as the orientation of the electric field with respect to individual members of the pair, will both be different from that assumed in our simple calculations above. Electric fields that are nonorthogonal to the chain axis of a molecule will have even stronger effects than found in our calculations, ${ }^{37}$ while the random arrangements of the molecule pairs with respect to the field in the experimental systems implies that the range of field over which a given spin channel dominates will be substantially larger than that found in our calculations. We therefore believe that a proper interpretation of our calculations is that in the experimental systems, there occur macroscopically large yields of both singlet and triplet excitons over a broad range of electric field. For small to moderate field strengths, the singlet channel dominates over the triplet channel. However, at still larger fields it is possible that this situation reverses. Whether or not this higher regime of field strength is experimentally accessible is a topic of future theoretical and experimental research.

\section{Chain-length dependence}

We now discuss the chain-length dependence of $\eta$ as has recently been determined experimentally. ${ }^{11,12}$ From careful measurements using different techniques, Wilson et al. ${ }^{11}$ and Wohlgenannt et al. ${ }^{12}$ have established that $\eta$ increases with conjugation length. Wilson et al. have shown that while $\eta$ is close to the statistically expected 0.25 in the monomer, ${ }^{11}$ it is substantially larger in the polymer. Wohlgenannt et al. have shown that $\sigma_{T} / \sigma_{S}$ increases linearly with the inverse of the conjugation length.

Within our numerical procedure, it is difficult to determine the chain length dependence of $\eta$ directly. This is because of multiple reasons, which include (i) the limitation to rather small sizes, (ii) the necessity to integrate $I_{m n}(t)$ over a complete period in each case in Figs. 3(a)-3(d) to obtain the total yield over that period, and (iii) the difference in the periods for singlet and triplet channels, as well as the differences among different singlet and triplet channels. We therefore present our discussion of chain-length dependence within a simplified formalism that is consistent with our time-dependent procedure.

Consider a transition (which could be of the chargetransfer type) between the states $|k\rangle$ and $|s\rangle$ of a two-state system, such that at time $t=0$ the system is in state $|s\rangle\left(c_{s}(0)=1, c_{k}(0)=0\right)$. We are interested in the yield $\left|c_{k}(t)\right|^{2}$ at a later time $t$ due to a perturbation $V_{k s}$. This is a standard textbook problem, ${ }^{30}$ and the time-dependent Schrödinger equation in this case is

$$
i \hbar \frac{\partial}{\partial t} c_{k}=V_{k s} \exp \left(i \omega_{k s} t\right) .
$$

For time-independent $V_{k s}$, as is true here $\left[V_{k s}\right.$ in the present case is simply $H_{\text {inter }}$ of Eq. (6)], the above equation is easily integrated to give

$$
\left|c_{k}(t)\right|^{2}=2|\langle k|V| s\rangle|^{2} \frac{1-\cos \omega_{k s} t}{\left(E_{k}^{(0)}-E_{s}^{(0)}\right)^{2}} .
$$

In our case $|s\rangle=\left|P_{1}^{+} P_{2}^{-}\right\rangle$, with the appropriate spin combinations, and $|k\rangle=\left|\left(1^{1} A_{g}\right)_{1}\left({ }^{1}{ }^{1} B_{u}\right)_{2}\right\rangle$ for the singlet channel, and $|k\rangle=\left|\left(1^{1} A_{g}\right)_{1}\left(1^{3} B_{u}\right)_{2}\right\rangle$ for the triplet channel (as usual, $|k\rangle$ can also have the chain indices reversed). We have already demonstrated (see Sec. III and Appendix A) that the matrix element $\langle k|V| s\rangle$ is nearly the same for the singlet and triplet channels, except near the unique point $X_{\perp} / t_{\perp}=0.5$. Ignoring the oscillation involving $\omega_{k s}$, we note that the relative yield of the singlet exciton is inversely proportional to the square of $\Delta E_{S}=\left[E\left(P^{+}\right)+E\left(P^{-}\right)-E\left(1^{1} A_{g}\right)\right.$ $\left.-E\left(1^{1} B_{u}\right)\right]$, while that of the triplet exciton is inversely proportional to the square of $\Delta E_{T}$, in which $E\left(1{ }^{1} B_{u}\right)$ in the above is replaced with $E\left(1{ }^{3} B_{u}\right)$ (note, however, that within the two-state approximation we have assumed that the singlet and triplet states that are of interest are the lowest singlet and triplet states; this can only be justified by the complete many-state calculations of the previous subsections). We see immediately that this simple two-state formalism predicts a higher singlet yield, since $E\left(1^{1} B_{u}\right)$ is considerably higher than $E\left(1^{3} B_{u}\right)$. Importantly, the chain-length dependence of $\eta$ is also understood from the above. Both $\Delta E_{S}$ and $\Delta E_{T}$ decrease with increasing chain length. However, the ratio $\Delta E_{S} / \Delta E_{T}$ also decreases, because of the covalent character of the $1{ }^{3} B_{u}$ and the ionic character of the $1{ }^{1} B_{u}$. This is seen most easily in the limit of the simple Hubbard model for the individual chains [zero intersite Coulomb interaction and 


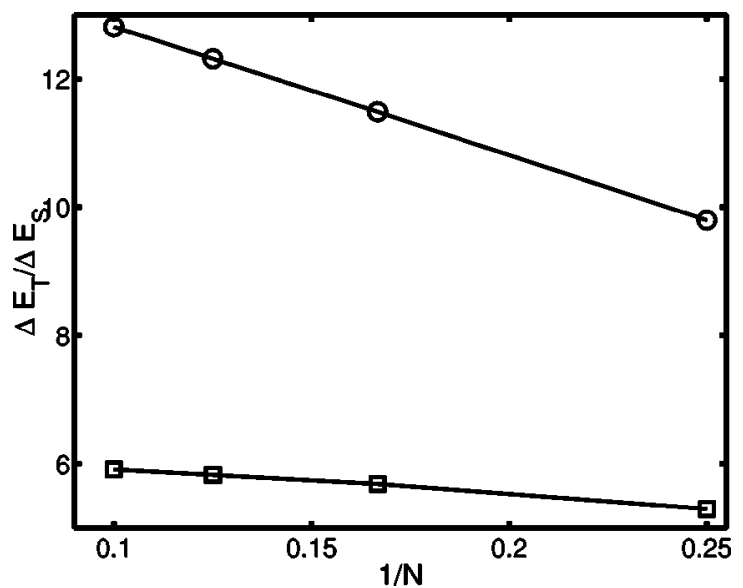

FIG. 9. $\Delta E_{T} / \Delta E_{S}$ vs $1 / N$ for the case of linear chains with ' ' $U-V$ ' ' extended Hubbard model for the case of (i) $U=5 \mathrm{eV}$ and $V=2 \mathrm{eV}$ (squares) and (ii) $U=6 \mathrm{eV}$, and $V=2 \mathrm{eV}$ (circles).

zero bond alternation in Eq. (4)], where $\Delta E_{S}$ approaches 0 and $\Delta E_{T}$ approaches $U$ in the long chain limit.

We have calculated $\Delta E_{S}$ and $\Delta E_{T}$ exactly for all chain lengths $N=4-10$ within the PPP-Ohno potential. While the ratio $\Delta E_{T} / \Delta E_{S}$ shows the correct qualitative trend (viz., increasing $\Delta E_{T} / \Delta E_{S}$ with increasing $N$ ) necessary for increasing $\eta$ with increasing $N$, the actual variation is small. This is to be expected, since our chain-length variation is small, and the Ohno potential decays very slowly. With our limitation on $N$, it is necessary that the Coulomb potential is short range, such that we have the same Hamiltonian at all chain lengths, as is approximately true for the experimental systems investigated. ${ }^{11,12}$ We have therefore done exact calculations of $\Delta E_{S}$ and $\Delta E_{T}$ for the extended Hubbard Hamiltonian $\left[V_{i j}\right.$ in Eq. (4) limited to nearest neighbor interaction $V]$ with parameters $t_{i j}=1.08 t_{0}$ and $0.92 t_{0}$ for double and single bonds, $V / t_{0}=2$ and $U / t_{0}=5$ and 6. In Fig. 9 we show our calculated results for $\Delta E_{T} / \Delta E_{S}$ for the two cases, for different $N$. In both cases, increasing $\Delta E_{T} / \Delta E_{S}$ with increasing $N$ indicates larger $\eta$ for longer chain lengths. Energy convergences are faster with larger $U$, which explains the steeper behavior of $\Delta E_{T} / \Delta E_{S}$ for larger $U$, and gives additional support to our argument.

\section{E. Role of heteroatoms}

The experiments by Baldo et al. ${ }^{7}$ and Wilson et al. ${ }^{11}$ both indicate that in small molecular systems $\eta$ can be close to 0.25 . This is in contrast to our results for ethylene (see Fig. 3 ). for which $\eta$ is calculated to be substantially larger. One reason for this might be that the Coulomb correlation effects in thin film samples are smaller than within the PPP Hamiltonian due to intermolecular interactions. The dominant effect, however, is due to the heteroatoms in the molecules investigated by these authors, as we show below. Specifically, the site-energy (electronegativity) difference between the heteroatom and carbon atoms makes these systems closer to the Hückel limit and this is what decreases $\eta$.

In order to compare with the model polyene systems we consider pairs of $(\mathrm{CH}=\mathrm{N})_{2}$ in the following calculations.
The single chain Hamiltonian [Eq. (4)] is then modified as follows. ${ }^{40}$ The Hubbard $U$ for the nitrogen atoms, $U_{N}$ $=12.34 \mathrm{eV}$. The local chemical potential $z_{N}$ for nitrogen with a $\pi$ lone pair involved in conjugation is 2 . Finally, nitrogen has site energy $\epsilon=-2.96 \mathrm{eV}$ relative to that of the carbon atoms. There are two possible arrangements for the two chains in a parallel configuration: (i) a carbon (nitrogen) atom on one chain lying directly above a carbon (nitrogen) atom on the other, and (ii) a carbon atom on one chain lying above a nitrogen atom on the second chain. We have chosen arrangement (i); there is no fundamental reason for arrangement (ii) to have a very different $\eta$.

In Figs. 10(a) and 10(b) we have plotted the $I_{m n}(t)$ for the singlet and triplet channels, respectively, for the case of $X_{\perp}$ $=0$. Figures $10(\mathrm{c})$ and $10(\mathrm{~d})$ show the same for $X_{\perp}$ $=0.1 \mathrm{eV}$. The most important conclusion that emerges from these calculations is that the relative yields of triplets are substantially larger in the present case, so much so that $\eta$ can be even close to the statistical limit of 0.25 (note that there are three triplet channels and the figures show the results for only one of these). We believe that these results give a qualitative explanation of the observation of Baldo et al. ${ }^{7}$ Taken together with the chain length dependence of $\eta$, as found in the previous subsection, these results also explain qualitatively the observations by Wilson et al. ${ }^{11}$ since the same chain-length dependence found in the case of simple polyenes should be true here also, although it is conceivable that rate of increase of $\eta$ with $N$ here may be slower.

\section{DISCUSSIONS AND CONCLUSIONS}

With a parallel arrangement of two polyene chains, we have shown that several experimentally observed qualitative features of the singlet-to-triplet yield ratios in $\pi$-conjugated systems can be understood within a well-defined total Hamiltonian for the two-chain system. While our model systems are rather simple, our theoretical treatment of the chargetransfer process between the two chains is exact. We have given a full time-dependent approach to the interchain charge-transfer process, and have shown that in systems containing only carbon atoms, the overall yield of the singlet exciton is considerably larger than that of triplet excitons and $\eta>0.25$. This is a direct consequence of moderate electronelectron Coulomb interactions which has strong effects on both the energies and the wave functions of the singlet and triplet excitons. The mechanism of the exciton yields that emerges from our calculations is as follows. For large yields, it is essential that there exist excited states of the composite two-chain system whose wave functions have simultaneously large overlaps with the wave function of the initial state consisting of polaron pairs, and the final state consisting of the two chains in the neutral states. Overlaps of the excited states of the two-chain system with final states in the singlet channel are considerably larger than for final states in the triplet channel, and this is what gives a large yield for the singlet exciton. This is a consequence of the different natures of the singlet and triplet excitons, which are ionic and covalent, respectively, in the VB notation. Our result here is consistent with experiments on long oligomers and 

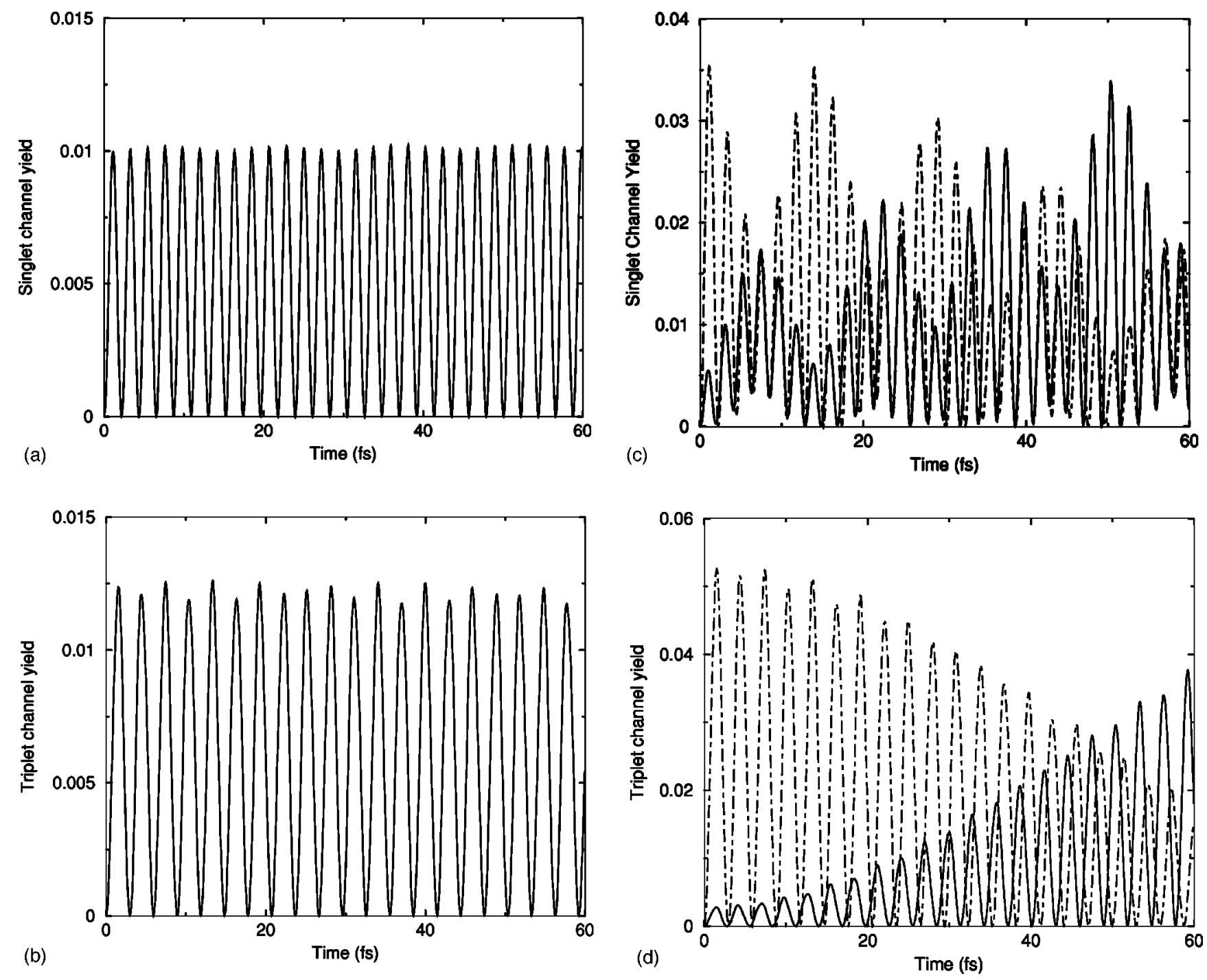

FIG. 10. Yields in the PPP model for the $(\mathrm{CH}=\mathrm{N})_{2}$ system. (a) Singlet and (b) triplet channels with $X_{\perp}=0$; (c) and (d) singlet and triplet channels with $X_{\perp}=0.1 \mathrm{eV}$. The state to which the yield is significant in (a) is $\left|S_{0} S_{1}\right\rangle$ while in (b) it is to the state $\left|S_{0} T\right\rangle$. The yield to states $\left|S_{1} S_{0}\right\rangle$ in singlet channel and $\left|T S_{0}\right\rangle$ in triplet channel are identical to those for $\left|S_{0} S_{1}\right\rangle$ and $\left|S_{0} T\right\rangle$ in (a) and (b), respectively. In (c) the yields to $\left|S_{1} S_{0}\right\rangle$ and $\left|S_{0} S_{1}\right\rangle$ are not the same and are shown separately. Similarly, in (d) yields to $\left|T S_{0}\right\rangle$ and $\left|S_{0} T\right\rangle$ are shown separately.

polymers. ${ }^{8-12}$ Although our exact calculations are limited to short chains, within a two-state approximation that is consistent with the full multilevel calculation we have shown that $\eta$ increases with the chain length, in agreement with experimental observations. ${ }^{11,12}$ The two-state approximation gives an alternate explanation of the higher yield of the singlet exciton that is related to the singlet and triplet exciton binding energies, which are substantially different in $\pi$-conjugated polymers. Finally, we have examined the role of heteroatoms, and have shown that in small molecular systems with nitrogen as the heteroatom, $\eta$ is substantially smaller, and may be even close to the statistically expected value of 0.25 . The wave functions in this case, due to the strong electronegativity difference between the heteroatom and carbon atoms, are closer to the Hückel limit, and this is what increases the relative yield of the triplet exciton. Our results here successfully explain the difference between $\mathrm{Alq}_{3}$ (Ref. 7) and heteroatom containing monomers ${ }^{11}$ on the one hand, and polymeric systems on the other, and thereby provide additional strong support to our theoretical approach.

The time-dependent approach to the charge-transfer process developed here is completely general and can be applied to many other similar processes, for example, photoinduced charge-transfer, triplet-triplet collisions in OLED's, etc. These and other applications are currently being investigated. Similarly, for a more complete understanding of the chain-length dependence of $\eta$, we will investigate chargetransfer process within the density matrix renormalization group technique.

Note added. Recently while this manuscript was under preparation we received a paper ${ }^{42}$ that discusses the relative yields of singlet and triplet excitons within the context of intrachain processes (see Sec. III) as opposed to the interchain process discussed here. Although the approach of these authors is different from ours, they also find that the relative yields of singlet and triplet excitons are determined by their 
binding energies (smaller binding energies giving larger yields). It is not clear whether the approach used by these authors applies to molecule-based OLED's. These authors have also investigated the effect of broken electron-hole symmetry, which is related to our calculations on chains of $(\mathrm{CH}=\mathrm{N})_{2}$. Our results here are different. While Karabunarliev and Bittner find even higher relative yield of singlet excitons (compared to electron-hole symmetric case) we find that $\eta$ here is smaller (see above). While a complete analysis of the electron-hole recombination must include both interchain and intrachain processes (and is a subject of future work in this area), we believe that this last result, when compared to experiments, justify our basic assumption that spin dependence of the yields of excitons can be understood largely within the context of intermolecular and interchain charge transfer.

\section{ACKNOWLEDGMENTS}

Work in Bangalore was supported by the CSIR, India and DST, India, through /INT/US (NSF-RP078)/2001. Work in Arizona was supported by NSF-DMR-0101659 and NSFINT-0138051. We are grateful to our experimental colleagues Z.V. Vardeny and M. Wohlgenannt for numerous stimulating discussions. S.M. acknowledges the hospitality of the Indian Institute of Science, Bangalore, where this work was completed.

\section{APPENDIX A}

We present here detailed analytic calculations of the matrix elements of $H_{\text {inter }}$ for the case of two ethylenes. We believe that these calculations give clear understandings of the chain-length independence of the calculated $\sigma_{S} / \sigma_{T}$ within the Fermi golden rule approach [Eq. (8)] that was presented in Sec. III (see Fig. 1). We also believe that even as these calculations show the inadequacy of the golden rule approach they provide an indirect understanding of the actual mechanism behind large $\eta$ in long-chain polymers.

As in the rest of the paper we consider parallel arrangements of the ethylene molecules, with sites 1 and 2 (3 and 4) corresponding to the lower (upper) molecule. Subscripts 1 and 2 that are assigned to wave functions describe the lower and upper molecule, respectively. The relevant singlemolecule eigenstates, corresponding to the lower molecule, then can be written as

$$
\begin{aligned}
&\left|1{ }^{1} A_{g}\right\rangle_{1}=\left(c_{1} / \sqrt{2}\right)\left(a_{1, \uparrow}^{\dagger} a_{1, \downarrow}^{\dagger}+a_{2, \uparrow}^{\dagger} a_{2, \downarrow}^{\dagger}\right)|0\rangle \\
&+\left(c_{2} / \sqrt{2}\right)\left(a_{1, \uparrow}^{\dagger} a_{2, \downarrow}^{\dagger}-a_{1, \downarrow}^{\dagger} a_{2, \uparrow}^{\dagger}\right)|0\rangle, \\
&\left|1{ }^{1} B_{u}\right\rangle_{1}=(1 / \sqrt{2})\left(a_{1, \uparrow}^{\dagger} a_{1, \downarrow}^{\dagger}-a_{2, \uparrow}^{\dagger} a_{2, \downarrow}^{\dagger}\right)|0\rangle, \\
&\left|{ }^{3} B_{u}\right\rangle_{1}=(1 / \sqrt{2})\left(a_{1, \uparrow}^{\dagger} a_{2, \downarrow}^{\dagger}+a_{1, \downarrow}^{\dagger} a_{2, \uparrow}^{\dagger}\right)|0\rangle, \\
&\left|P^{+}\right\rangle_{1}=(1 / \sqrt{2})\left(a_{1, \sigma}^{\dagger}+a_{2, \sigma}^{\dagger}\right)|0\rangle, \\
&\left|P^{-}\right\rangle_{1}=(1 / \sqrt{2})\left(a_{1, \uparrow}^{\dagger} a_{1, \downarrow}^{\dagger} a_{2, \sigma}^{\dagger}-a_{2, \uparrow}^{\dagger} a_{2, \downarrow}^{\dagger} a_{1, \sigma}^{\dagger}\right)|0\rangle .
\end{aligned}
$$

In the above $|0\rangle$ is the vacuum for chain 1 and $c_{1}$ and $c_{2}$ are the coefficients of the ionic and covalent configurations in the $1 A_{g}$ ground state that are to be determined by solving for the $2 \times 2 A_{g}$ subspace of ethylene within the PPP Hamiltonian $\left[c_{1}=c_{2}=1 / \sqrt{2}\right.$ in the Hückel Hamiltonian and the matrix elements in Eq. (8) in the singlet and triplet channels are exactly equal in this case]. We have chosen the $M_{S}=0$ wave function for the $1^{3} B_{u}$, but what follows is equally true for the $M_{S}= \pm 1$ wave functions. We have not assigned definite spin states to the charged polaronic wave functions, since the charged molecules can have either spin, and since different combinations of these spin states give the initial spin singlet or triplet product eigenstates for $\left|P^{+} P^{-}\right\rangle$. Note, however, the relative minus signs between the two configurations in $\left|P^{-}\right\rangle$, as opposed to the relative plus signs between the two configurations in $\left|P^{+}\right\rangle$. This is what ensures that the product wave functions of the type $\left|P_{1}^{+} P_{2}^{-}\right\rangle$, with positive charge on molecule 1 and negative charge on molecule 2 , has odd parity with respect to the center of inversion on a single chain, and charge recombination can therefore only generate neutral states that have odd parity (for example, $\left|1{ }^{1} A_{g}\right\rangle_{1}\left|1{ }^{1} B_{u}\right\rangle_{2}$ but not $\left.\left|1{ }^{1} A_{g}\right\rangle_{1}\left|2{ }^{1} A_{g}\right\rangle_{2}\right)$.

We consider the initial states $\left|i_{S}\right\rangle$ and $\left|i_{T}\right\rangle$ first, which are constructed from taking products of the polaronic wave functions given above. Since these product functions contain four terms each, and also since one of our goals is to arrive at a visual representation of the charge recombination process in configuration space, we have chosen not to write their explicit form but have given in Fig. 11 the wave functions in the VB notation, where a singlet bond between sites $i$ and $j$ is defined as $2^{-1 / 2}\left(a_{i, \uparrow}^{\dagger} a \dagger_{j, \downarrow}-a_{i, \downarrow}^{\dagger} a \dagger_{j, \uparrow}\right)|0\rangle$, a triplet bond (with an arrow pointing from site $i$ to site $j$ ) is defined as $2^{-1 / 2}\left(a_{i, \uparrow}^{\dagger} a \dagger_{j, \downarrow}+a_{i, \downarrow}^{\dagger} a \dagger_{j, \uparrow}\right)|0\rangle$, and crosses correspond to doubly occupied sites $a_{i, \uparrow}^{\uparrow} a \dagger_{j, \downarrow}|0\rangle$. Given the initial and final states, it is now easily seen that $V_{i, j}$ in $H_{\text {inter }}$ [Eq. (6)] plays no role within the golden rule approach, ${ }^{15,16}$ since this term causes no transition between the initial and final states (note, however, $V_{i, j}$ can play a significant role in the full dynamics calculation of Sec. IV). The matrix elements of the remaining terms in $H_{\text {inter }}$ are now readily evaluated and these are given below:

$$
\begin{aligned}
& \left\langle\left(1{ }^{1} A_{g}\right)_{1}\left(1{ }^{1} B_{u}\right)_{2}\left|H_{\text {inter }}\right| P_{1}^{+} P_{2}^{-}\right\rangle_{S} \\
& \quad=-\left(c_{1} / \sqrt{2}\right)\left(-t_{\perp}+2 X_{\perp}\right)-\left(c_{2} / \sqrt{2}\right)\left(-t_{\perp}+X_{\perp}\right),
\end{aligned}
$$

$$
\begin{aligned}
& \left\langle\left(1{ }^{1} A_{g}\right)_{2}\left(1{ }^{1} B_{u}\right)_{1}\left|H_{\text {inter }}\right| P_{1}^{+} P_{2}^{-}\right\rangle_{S} \\
& \quad=\left(c_{1} / \sqrt{2}\right)\left(-t_{\perp}+2 X_{\perp}\right)+\left(c_{2} / \sqrt{2}\right)\left(-t_{\perp}+3 X_{\perp}\right),
\end{aligned}
$$

$$
\begin{aligned}
& \left\langle\left(1^{1} A_{g}\right)_{1}\left(1^{3} B_{u}\right)_{2}\left|H_{\text {inter }}\right| P_{1}^{+} P_{2}^{-}\right\rangle_{T} \\
& \quad=-\left(c_{1} / \sqrt{2}\right)\left(-t_{\perp}+X_{\perp}\right)-\left(c_{2} / \sqrt{2}\right)\left(-t_{\perp}+2 X_{\perp}\right),
\end{aligned}
$$


(a)
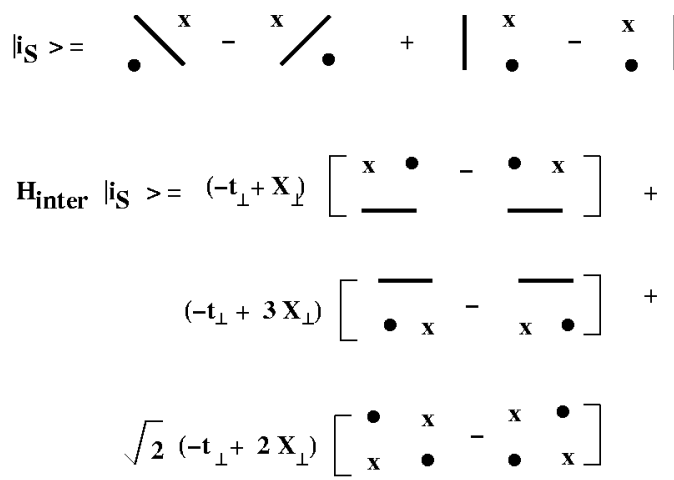

(b)

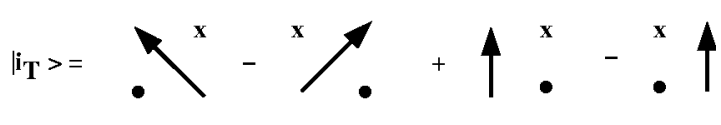

$\mathbf{H}_{\text {inter }} \mid \mathbf{i}_{\mathbf{T}}>=\left(-\mathrm{t}_{\perp}+\mathbf{X}_{\perp}\right)\left[\begin{array}{llll}\bullet & \mathbf{x} & + & \mathbf{x} \\ \longrightarrow\end{array}\right]+$

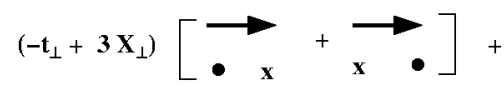

$\sqrt{2}\left(-\mathrm{t}_{\perp}+2 \mathrm{x}_{\perp}\right)[\boldsymbol{A}|-| \boldsymbol{A}]$

(c)

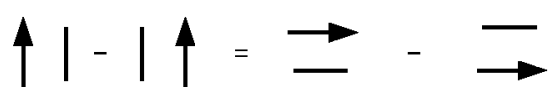

FIG. 11. The initial (a) singlet and (b) triplet states $\left|P_{1}^{+} P_{2}^{-}\right\rangle$for the case of two ethylenes, and the result of operating with $H_{\text {inter }}$. The upper (lower) two sites correspond to molecule 1 (molecule 2). The result (c) is a linear relationship between covalent triplet VB diagrams.

$$
\begin{aligned}
& \left\langle\left(1{ }^{1} A_{g}\right)_{1}\left(1{ }^{3} B_{u}\right)_{2}\left|H_{\text {inter }}\right| P_{1}^{+} P_{2}^{-}\right\rangle_{T} \\
& \quad=\left(c_{1} / \sqrt{2}\right)\left(-t_{\perp}+3 X_{\perp}\right)+\left(c_{2} / \sqrt{2}\right)\left(-t_{\perp}+2 X_{\perp}\right) .
\end{aligned}
$$

Several points are to be noted now. First, for $X_{\perp}=0$, the squares of all the matrix elements are equal, and hence there is no difference between singlet and triplet generation within the golden rule approach in this limit, and we agree on this with Shuai et al. ${ }^{15}$ Second, however, defining overall $\sigma_{S}$ as the sum of the squares of the matrix elements in Eqs. (A2a) and (A2b), and $\sigma_{T}$ as the sum of the squares of the matrix elements in Eqs. (A2c) and (A2d), respectively, we see that $\sigma_{S} / \sigma_{T}$ depends very weakly on $X_{\perp} / t_{\perp}$ at all $X_{\perp} / t_{\perp}$ except for $X_{\perp} / t_{\perp}$ very close to 0.5 , where $-t_{\perp}+2 X_{\perp}=0$ and $-t_{\perp}+X_{\perp}$ and $-t_{\perp}+3 X_{\perp}$ have opposite signs. This is particularly so for the calculated $c_{1}$ and $c_{2}$ for PPP-Ohno parameters $\left(c_{1}=0.5786, c_{2}=0.8156\right)$. We now examine the different terms in Eqs. (A2a)-(A2d) in detail. From Fig. 11 we note that there are three classes of interchain electron transfers: (i) charge transfer between sites that are both singly occupied, leading to a doubly occupied site or an empty site (denoted by $1+1 \rightarrow 2+0$, where the numbers denote site occupancies)—or the exact reverse process, (ii) charge transfers of the type $1+0 \rightarrow 0+1$, using the same notation, and (iii) charge transfers of the type $2+1 \rightarrow 1+2$, again with the same notation. These three processes have different matrix elements $\left(-t_{\perp}+2 X_{\perp}\right),\left(-t_{\perp}+X_{\perp}\right)$, and $\left(-t_{\perp}+3 X_{\perp}\right)$, respectively. The role of $X_{\perp}$ now becomes absolutely clear. Nonzero $X_{\perp}$ creates an asymmetry between the upper and lower molecule, leading to a difference between the yields of $\left|1{ }^{1} A_{g}\right\rangle_{1}\left|1{ }^{1} B_{u}\right\rangle_{2}$ and $\left|1{ }^{1} A_{g}\right\rangle_{2}\left|1{ }^{1} B_{u}\right\rangle_{1}$, but it does not create a significant difference between $\sigma_{S}$ and $\sigma_{T}$.

At exactly $X_{\perp} / t_{\perp}=0.5$ terms containing $\left(-t_{\perp}+2 X_{\perp}\right)$ in the matrix elements vanish, while the other terms are also small and of opposite signs. The singlet channel matrix elements now involve only $c_{2}$, while the triplet channel matrix elements involve only $c_{1}$. Since for repulsive Coulomb interactions $c_{2}>c_{1}$, the sum of the the squares of the matrix elements here are larger for the singlet channel than for the triplet channel. This is what is reflected in our plot of Fig. 8 . Note, however, that the calculated yields approach zero in both cases here. It is also clear from Eqs. (17) that this difference between the singlet and triplet channels persist over a very narrow region about $X_{\perp} / t_{\perp}=0.5$. We, therefore, do not believe that this is of any relevance for realistic systems.

Our final point concerns the chain-length independence of our results in Fig. 8 (except near $X_{\perp} / t_{\perp}=0.5$ ). For arbitrary chain lengths there can occur only the three classes of interchain charge transfers discussed above $(1+1 \rightarrow 2+0,1+0$ $\rightarrow 0+1$, and $2+1 \rightarrow 1+2$ ). The detailed wave functions of longer chains are different, but the expectation values $\left\langle n_{i, \uparrow} n_{i, \downarrow}\right\rangle$ for the different wave functions are nearly the same for fixed intrachain correlation parameters. Thus although in long chains there can in principle occur many more interchain hops that are of the type $1+1 \rightarrow 2+0$, such charge transfers lead to additional double occupancies (relative to the overall initial states) that are energetically costly because of electron correlation effects. Such charge transfers therefore make weak contributions to the overall interchain charge transfer. The net consequence is the weak chainlength dependence found in Fig. 1 at all points other than $X_{\perp} / t_{\perp}=0.5$.

We believe that the above detailed calculation, aside from indicating the inapplicability of the golden rule, also indicates that the proper theoretical treatment must include the differences in the energies and wave functions of the final states, as indeed is done in our time-dependent calculations.

\section{APPENDIX B}

Details of the numerical procedure that were not discussed in Sec. V are given below. The charged as well as neutral eigenstates of $H_{\text {intra }}$ for individual chains are obtained in the VB representation by using a diagrammatic VB approach $^{28}$ with bit representation of the basis states. The eigenstates of a given spin $S$ are obtained for $M_{S}=S$. The VB eigenstates are then transformed to the basis of Slater determinants with $M_{S}=S$ by expanding the terms in each singlet pair and assigning an up-spin at each unpaired site with single occupancy. Thus, a triplet VB basis consisting of two singlet pairs and corresponding to a function with $M_{S}$ 
$=1$ is expanded into four Slater determinants each with $M_{S}$ $=1$. To obtain eigenstate corresponding to other $M_{S}$ values with Slater determinantal basis, we apply the $\hat{S}^{-}$operator on the state, as many times as is necessary.

We use the eigenstates of $\left|P^{+}\right\rangle$and $\left|P^{-}\right\rangle$to form the initial state of chosen spin in the form,

$$
\begin{aligned}
\Psi_{1,0}(0)= & \frac{1}{\sqrt{2}}\left[\left|\frac{1}{2},+\frac{1}{2}\right\rangle_{1} \times\left|\frac{1}{2},-\frac{1}{2}\right\rangle_{2}\right. \\
& \left. \pm\left|\frac{1}{2},-\frac{1}{2}\right\rangle_{1} \times\left|\frac{1}{2},+\frac{1}{2}\right\rangle_{2}\right],
\end{aligned}
$$

where the subscripts 1 and 0 refer to the total spin of the initial state. The direct product of the states are expressed in the Slater determinantal basis of the composite system with the coefficient $c_{k}$ of the basis state $|k\rangle$ in the composite system being given by

$$
c_{k}=\sum_{l, m} d_{l} d_{m}\langle k \mid l \times m\rangle,
$$

where $d_{l}$ and $d_{m}$ are the coefficients of the basis states $|l\rangle$ and $|m\rangle$ in the ground states of the subsystems 1 and 2, respectively. The direct product itself is effected by shifting the $2 n_{1}$ bits of the integer representing the basis state of system 1 with $n_{1}$ sites to the immediate left of the $2 n_{2}$ bits in the integer that represents the basis state of system 2 with $n_{2}$ sites. The resulting larger integer with $2\left(n_{1}+n_{2}\right)$ bits correspond to an integer that represents one of the basis states of the composite system of $\left(n_{1}+n_{2}\right)$ sites.

The evolution of the initial state involves solving the linear algebraic equations, $A \mathbf{x}(t+\Delta t)=\mathbf{b}$, where the matrix elements of the matrix $A$ and the components of $\mathbf{b}$ are given by

$$
\begin{gathered}
A_{i j}=\left(\delta_{i j}+i \hbar H_{i j} \frac{\Delta t}{2}\right), \\
b_{i}=\sum_{j}\left(\delta_{i j}-i \hbar H_{i j} \frac{\Delta t}{2}\right) x_{j}(t) .
\end{gathered}
$$

The matrix $A$ in the largest system we have studied is nearly of order one million. For reasonable convergence of the solution of the system of equations we need a $\Delta t$ of the order of $0.05 \mathrm{eV} / \hbar$, which is typically $0.033 \mathrm{fs}$, and this guarantees diagonal dominance of the matrix $A$. Thus, if one wishes to follow the dynamics for even as long as say 60 femtoseconds one needs to solve the linear system about 2000 times. This is rendered possible by the sparseness of the matrix $A$. For efficient convergence, we use a small matrix algorithm ${ }^{41}$ which is very similar to the Davidson's algorithm for matrix eigenvalue problem. In the case of the largest system size, it takes about $12 \mathrm{~h}$ on a DEC Alpha $333 \mathrm{MHz}$ system to evolve the state by $60 \mathrm{fs}$ for a given channel.

At the end of each iteration in the evolution of the state, we obtain the intensity or the yield in a pair of states in the neutral subsystem. The number of such pairs is enormous when we deal with say two systems of six sites each. The number of pairs in the singlet channel is 30625 while that in the triplet channel is 33075 . We can reduce the pairs onto which we project the evolved state by restricting ourselves to a few low-energy states of the neutral subsystem. However, even in this case, the number of pairs could be rather large. To overcome this problem, we select only those pairs which have a minimum yield of say $10^{-4}$ at all times. This restriction when implemented judiciously leaves us with only a few pairs with significant yields.
*Present address: GE John F. Welch Technology Center, Sy 152, Export Promotion Industrial Park, Ph 2, Hoodi Village, White Field Road, Bangalore 560 066, India

${ }^{1}$ C. W. Tang and S. A. V. Slyke, Appl. Phys. Lett. 51, 913 (1987).

${ }^{2}$ J. H. Burroughes, D. D. C. Bradley, A. R. Brown, R. N. Marks, K. Mackay, R. H. Friend, P. L. Burns, and A. B. Holmes, Nature (London) 347, 539 (1990).

${ }^{3}$ G. Gustafsson, Y. Cao, G. M. Treacy, F. Klavetter, N. Colaneri, and A. Heeger, Nature (London) 357, 477 (1992).

${ }^{4}$ F. Garten, Alain Hilberer, F. Cacialli, E. Esselink, Y. van Dam, A. R. Schlatmann, R. Friend, T. M. Klapwijk, and G. Hadziioannou, Adv. Mater. 9, 127 (1997).

${ }^{5}$ R. H. Friend, R. W. Gymer, A. B. Holmes, J. H. Burroughes, R. N. Marks, C. Taliani, D. D. C. Bradley, D. A. Dos Santos, J. L. Brédas, M. Lögdlund, and W. R. Salaneck, Nature (London) 397, 121 (1999).

${ }^{6}$ N. S. Sariciftci, Curr. Opin. Solid State Mater. Sci. 4, 373 (1999).

${ }^{7}$ M. A. Baldo, D. F. O'Brien, M. E. Thompson, and S. R. Forrest, Phys. Rev. B 60, 14422 (1999).

${ }^{8}$ Y. Cao, I. D. Parker, G. Yu, C. Zhang, and A. J. Heeger, Nature (London) 397, 414 (1999).

${ }^{9}$ P. K. H. Ho, J. S. Kim, J. H. Burroughes, H. Becker, S. F. Y. Li, T. M. Brown, F. Cacialli, and R. H. Friend, Nature (London) 404, 481 (2000).
${ }^{10}$ M. Wohlgenannt, K. Tandon, S. Mazumdar, S. Ramasesha, and Z. V. Vardeny, Nature (London) 409, 494 (2001).

${ }^{11}$ J. S. Wilson, A. S. Dhoot, A. J. A. B. Seeley, M. S. Khan, A. Köhler, and R. H. Friend, Nature (London) 413, 828 (2001).

${ }^{12}$ M. Wohlgenannt, X. M. Jiang, Z. V. Vardeny, and R. A. J. Janssen, Phys. Rev. Lett. 88, 197401 (2002).

${ }^{13}$ T.-M. Hong and H.-F. Meng, Phys. Rev. B 63, 075206 (2001).

${ }^{14}$ M. N. Kobrak and E. R. Bittner, Phys. Rev. B 62, 11473 (2000).

${ }^{15}$ Z. Shuai, D. Beljonne, R. J. Silbey, and J. L. Bredas, Phys. Rev. Lett. 84, 131 (2000).

${ }^{16}$ A. Ye, Z. Shuai, and J. L. Bredas, Phys. Rev. B 65, 045208 (2002).

${ }^{17}$ A. P. Monkman, H. D. Burrows, M. da G. Miguel, I. Hamblett, and S. Navaratnam, Chem. Phys. Lett. 307, 303 (1999).

${ }^{18}$ A. P. Monkman, H. D. Burrows, L. J. Hartwell, L. E. Horsburgh, I. Hamblett, and S. Navaratnam, Phys. Rev. Lett. 86, 1358 (2001).

${ }^{19}$ R. Osterbacka, M. Wohlgenannt, D. Chinn, and Z. V. Vardeny, Phys. Rev. B 60, R11 253 (1999).

${ }^{20}$ Y. V. Romanovskii, A. Gerhard, B. Schweitzer, U. Scherf, R. I. Personov, and H. Bässler, Phys. Rev. Lett. 84, 1027 (2000).

${ }^{21}$ D. Beljonne, Z. Shuai, R. H. Friend, and J. L. Bredas, J. Chem. Phys. 102, 2042 (1995). 
${ }^{22}$ M. Chandross and S. Mazumdar, Phys. Rev. B 55, 1497 (1997).

${ }^{23}$ R. Pariser and R. G. Parr, J. Chem. Phys. 21, 466 (1953).

${ }^{24}$ J. A. Pople, Trans. Faraday Soc. 49, 1375 (1953).

${ }^{25}$ K. Ohno, Theor. Chim. Acta 2, 219 (1964).

${ }^{26}$ D. K. Campbell, J. T. Gammel, and E. Y. Loh Jr., Phys. Rev. B 42, 475 (1990).

${ }^{27}$ M. J. Rice and Y. N. Gartstein, Phys. Rev. B 53, 10764 (1996).

${ }^{28}$ Z. G. Soos and S. Ramasesha, Phys. Rev. B 29, 5410 (1984).

${ }^{29}$ P. Tavan and K. Schulten, Phys. Rev. B 36, 4337 (1987).

${ }^{30}$ E. Merzbacher, in Quantum Mechanics (Wiley, New York, 1970).

${ }^{31}$ C. Kittel, Quantum Theory of Solids (Wiley, New York, 1963), Chap. 6.

${ }^{32}$ A. Messiah, in Quantum Mechanics (North-Holland, Amsterdam, 1972).

${ }^{33}$ J. Crank and P. Nicholson, Proc. Cambridge Philos. Soc. 43, 50 (1947).
${ }^{34}$ R. Varga, Matrix Iterative Analysis (Prentice-Hall Inc., New Jersey, 1962).

${ }^{35}$ I. I. Rabi, Phys. Rev. 51, 652 (1937).

${ }^{36}$ L. Allen and J. H. Eberly, Optical Resonance and Two Level Atoms (Dover, New York, 1987).

${ }^{37}$ D. Guo, S. Mazumdar, S. N. Dixit, F. Kalzar, F. Jarka, Y. Kawabe, and N. Peyghambarian, Phys. Rev. B 48, 1433 (1993).

${ }^{38}$ G. Weiser, Phys. Rev. B 45, 14076 (1992).

${ }^{39}$ M. Liess, S. Jeglinski, Z. V. Vardeny, M. Ozaki, K. Yoshino, Y. Ding, and T. Barton, Phys. Rev. B 56, 15712 (1997).

${ }^{40}$ S. Ramasesha, I. D. L. Albert, and P. K. Das, Phys. Rev. B 43, 7013 (1991).

${ }^{41}$ S. Rettrup, J. Comput. Phys. 45, 100 (1982).

${ }^{42}$ S. Karabunarliev and E. R. Bittner, cond-mat/0206015 (unpublished). 